Technische Universität IImenau

Institut für Volkswirtschaftslehre

Diskussionspapier Nr. 136

Die Theorie mehrseitiger Marktplätze in der US-amerikanischen und deutschen Zusammenschlusskontrolle: Eine empirische Untersuchung für den Mediensektor

Heidi Dittmann \& Björn A. Kuchinke

Januar 2020

Institut für Volkswirtschaftslehre

Ehrenbergstraße 29

Ernst-Abbe-Zentrum

D-98 684 Ilmenau

Phone 03677/69-4030/-4032

Fax 03677/69-4203

https://www.tu-ilmenau.de/wpo/forschung/

ISSN 0949-3859 


\title{
Die Theorie mehrseitiger Marktplätze in der US-amerikanischen und deutschen Zusammenschlusskontrolle: Eine empirische Untersuchung für den Mediensektor
}

\author{
Heidi Dittmann`, Björn A. Kuchinke^
}

\section{Inhaltsverzeichnis}

$1 \quad$ Einleitung

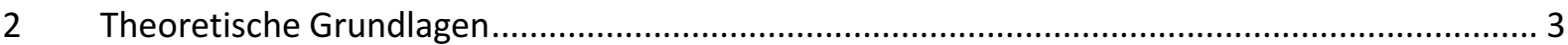

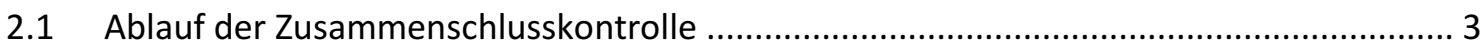

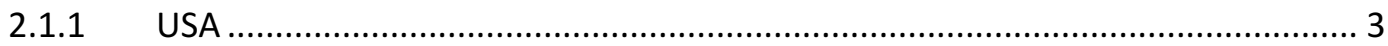

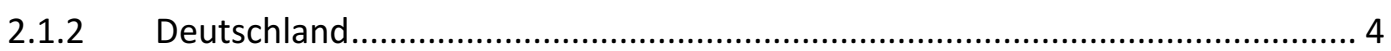

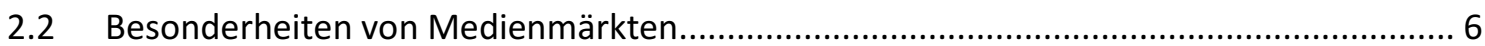

2.3 Implikationen für die Zusammenschlusskontrolle................................................... 7

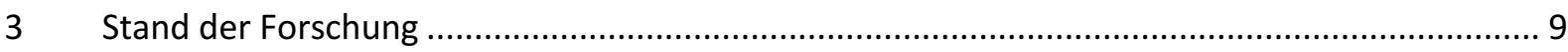

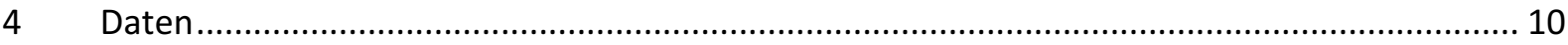

4.1 USA

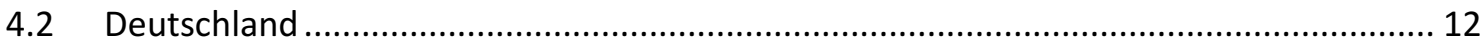

5 Analyse

5.1 USA

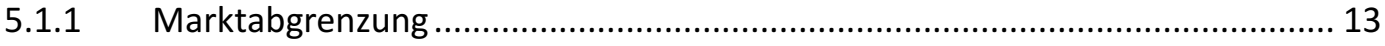

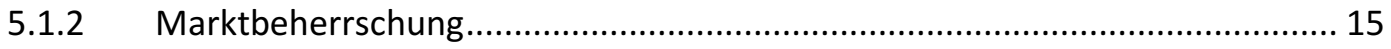

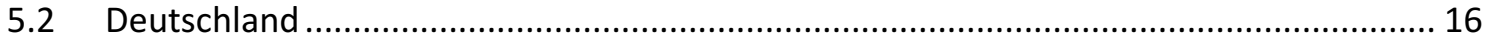

5.2.1 Marktabgrenzung ......................................................................... 16

5.5.2 Marktbeherrschung............................................................................. 18

5.3 Vergleichende Gesamtbetrachtung ........................................................... 19

5.4 Kritische Reflexion ................................................................................... 22

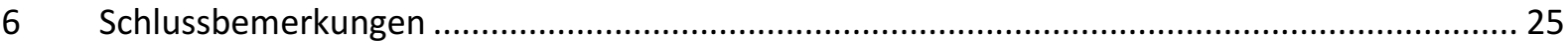

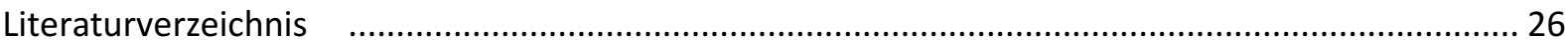

- Dr. Heidi Dittmann, M. Sc., Bauhaus-Universität Weimar, Fakultät Medien, Professur Medienökonomik, Albrecht-Dürer-Str. 2, 99425 Weimar.

- Prof. Dr. Björn A. Kuchinke, Bauhaus-Universität Weimar, Fakultät Medien, Professur Medienökonomik, Albrecht-Dürer-Str. 2, 99425 Weimar, Mail: bjoern.kuchinke@uni-weimar.de. 
Die grundlegende Zielsetzung der Zusammenschlusskontrolle besteht in der Aufrechterhaltung einer Marktstruktur, die wirksamen Wettbewerb zulässt. ${ }^{1} \mathrm{Zu}$ untersagen sind Zusammenschlüsse immer dann, wenn sie zu einer Entstehung oder Verstärkung einer marktbeherrschenden Stellung führen, die den beteiligten Unternehmen die Möglichkeit eröffnet, Preise oberhalb des Wettbewerbspreises zu setzen. Bei Medienunternehmen handelt es sich jedoch häufig um Plattformen im Sinne der Theorie mehrseitiger Marktplätze, die eine Reihe von Besonderheiten gegenüber klassischen Unternehmen aufweisen. Zur bestmöglichen Internalisierung der zwischen den Nachfragegruppen bestehenden indirekten Netzeffekte wählen Plattformen eine von traditionellen Märkten abweichende Preisstruktur. ${ }^{2}$ Da der Nutzen der Akteursgruppen mit steigender Gruppengröße zunimmt, weisen Plattformen eine gewisse Tendenz zur Konzentration auf. ${ }^{3}$ Marktmacht oder gar Monopolstellungen müssen auf mehrseitigen Märkten jedoch nicht zwingend mit negativen Wohlfahrtseffekten einhergehen. Vielmehr können sie sogar wohlfahrtsoptimal sein. ${ }^{4}$ Werden diese Besonderheiten im Rahmen der Zusammenschlusskontrolle nicht gewürdigt, kann es zu Fehlentscheidungen kommen. Insbesondere besteht die Gefahr der Untersagung von Zusammenschlussvorhaben, von denen positive Wohlfahrtseffekte ausgehen könnten.

Die Theorie mehrseitiger Marktplätze hat sich seit den ersten Arbeiten von Rochet/Tirole (2003), Evans (2003) und Evans/Schmalensee (2007) und ihrer Übertragung auf Medienmärkte durch Dewenter (2006) und Dewenter/Haucap (2009) zur Analyse von Plattformmärkten im wissenschaftlichen Diskurs inzwischen etabliert. Die Würdigung der plattformspezifischen Besonderheiten war bisher weder im US-amerikanischen noch im deutschen Kartellrecht verpflichtend vorgegeben. Mit der 9. GWB-Novelle wurden diese erstmalig in das deutsche Kartellrecht aufgenommen. Vor dem Hintergrund der aufgezeigten Gefahren bei der Vernachlässigung dieser besteht das Ziel des vorliegenden Beitrags in der Untersuchung der praktischen Relevanz der theoretischen Erkenntnisse in der Zusammenschlussprüfung der US-

1 Mögliche Auswirkungen von Medienfusionen auf die Meinungsvielfalt werden nachfolgend nicht betrachtet. Hierfür wird auf die Ausführungen von Dewenter (2007), Anderson/McLaren (2012) und Budzinski (2009) verwiesen.

2 Vgl. Rochet/Tirole, 2003 S. 990, Schmalensee, 2002, S. 105, Evans/Schmalensee, 2007, S. 154.

3 Ausführlicher hierzu vgl. Haucap/Wenzel, 2009, S. $14 \mathrm{ff}$.

4 Vgl. Weisman, 2005, S. 137, Klein et al., 2006, S. 595, Dewenter/Haucap/Wenzel, 2011, S. 13 ff. Diese Überlegungen werden aufgegriffen in Lindstädt, 2009, S. 22. 
amerikanischen und deutschen Kartellbehörden. Die Analyse gliedert sich wie folgt: Nach einer Beschreibung der theoretischen Grundlagen (Kapitel 2) sowie der Darlegung des Stands der Forschung (Kapitel 3) werden die für die Untersuchung erhobenen Daten beschrieben (Kapitel 4). Die vergleichende Analyse ist Gegenstand des fünften Kapitels. Der Beitrag schließt mit Schlussbemerkungen in Kapitel 6.

\section{Theoretische Grundlagen}

\subsection{Ablauf der Zusammenschlusskontrolle}

\subsubsection{USA}

Das amerikanische Kartellrecht setzt sich im Wesentlichen aus den Regelungen des Sherman Acts von 1890 und des Clayton Acts von 1914 zusammen. Nach $\S 1$ und 2 ShAct sind sämtliche horizontale und vertikale Wettbewerbsbeschränkungen ebenso verboten wie der Versuch der oder die tatsächlich erreichte Monopolisierung. Unternehmenszusammenschlüsse sind nach $\S 7 \mathrm{ClAct}$ insbesondere zu untersagen, wenn sie zu einer spürbaren Beschränkung des Wettbewerbs oder zur Monopolisierung führen. Davon umfasst sind sowohl der Erwerb von Geschäftsanteilen als auch der Erwerb von Vermögensgegenständen. Während die Erlangung einer marktbeherrschenden Stellung durch internes Wachstum nicht verhindert werden kann, soll externes Wachstum unterbunden werden, wenn es wettbewerbsschädliche Wirkungen entfaltet. Zu diesem Zweck sind alle Zusammenschlussvorhaben von Unternehmen, welche die in § 7A ClAct vorgegebenen Schwellenwerte hinsichtlich ihres Nettoumsatzes oder ihrer Bilanzsumme überschreiten, anmeldepflichtig. Die Zuständigkeit liegt gemäß § 11a und § 15 ClAct bei der Federal Trade Commission (FTC) sowie bei der Antitrust Division (ATD) des Departments of Justice (DoJ). Prinzipiell sind beide Institutionen zwar in ihren Entscheidungen unabhängig, ein gewisser indirekter politischer Einfluss kann durch die Bestimmung des Leiters der ATD, der Mitglieder der FTC sowie der Richter des Supreme Courts als letzte Verfahrensinstanz durch den Präsidenten und den Kongress indes nicht ausgeschlossen werden.

Die Beurteilung der Zusammenschlussvorhaben erfolgt im Wesentlichen nach den Vorgaben der Horizontal und der Non-Horizontal Merger Guidelines. Zur Definition des relevanten Markts stellen FTC und ATD im Wesentlichen auf den SSNIP-Test ab. Die Marktzugehörigkeit von Gütern und Dienstleistungen bestimmt sich dabei über die Reaktion der Verbraucher auf Preisänderungen. Gelingt es dem hypothetischen Monopolisten, den Preis seines Produkts A 
für einen nicht nur vorübergehenden Zeitraum signifikant zu erhöhen, ohne signifikante Abwanderungen der Nachfrage zu Produkt B zu bewirken, werden beide Produkte vom Verbraucher nicht als Substitute erachtet und gehören daher nicht zum gleichen Markt. Findet eine Substitution von A durch B statt, handelt es sich um einen gemeinsamen Markt. ${ }^{5}$

Zusammenschlüsse sind zu untersagen, wenn es zu einer Erhöhung der Marktkonzentration, gemessen am HHI, und zudem zu negativen Wettbewerbseffekten kommt. Letztere werden angenommen, wenn mit dem Zusammenschluss die Wahrscheinlichkeit aufeinander abgestimmter Verhaltensweisen oder unilateraler Effekte steigt. ${ }^{6}$ Als verbotsabwendenden Einwand sieht das US-amerikanische Kartellrecht seit 1984 die Möglichkeit der Efficiency Defense vor. Können Unternehmen durch den Zusammenschluss entstehende Effizienzgewinne, wie technischer Fortschritt, Qualitätssteigerungen oder Serviceverbesserungen nachweisen, kann eine Untersagung abgewendet werden. ${ }^{7}$ Für vertikale und konglomerate Fusionen sind die Grenzen für die Anerkennung der Effizienzeinreden dabei geringer als für Zusammenschlüsse von Wettbewerbern.

Weder der Sherman noch der Clayton Act oder die Merger Guidelines sehen medienspezifische Besonderheiten im Rahmen der Fusionskontrolle vor. Konkrete Bestrebungen zur Aufnahme der Theorie mehrseitiger Marktplätze lassen sich derzeit nicht beobachten. In der Kritik steht die kartellrechtliche Praxis im Wesentlichen aufgrund der hohen Konzentration auf einigen Märkten. ${ }^{8}$

\subsubsection{Deutschland}

Die deutsche Zusammenschlusskontrolle sieht eine Anmeldepflicht für Zusammenschlussvorhaben von Unternehmen, deren Umsätze die gesetzlich normierten Schwellenwerte des § 35 Abs. 1 GWB überschreiten, vor. Abzulehnen sind diese, wenn von einer erheblichen Behinderung des Wettbewerbs auszugehen ist ( $\$ 36$ Abs. $1 \mathrm{GWB}$ ). Die Prüfung der Zusammenschlussfälle erfolgt in erster Instanz durch das Bundeskartellamt, welches institutionell unabhängig

5 Zum Wesen des SSNIP-Tests vgl. Motta, 2004, S. 102 f., Europäische Kommission, Bekanntmachung über die Definition des relevanten Marktes im Sinne des Wettbewerbsrechts der Gemeinschaft, ABI. 1997 C 372/5, S. 6, Emch/Thompson, 2006, 14 f., Filistrucchi, 2008, S. 5, Filistrucchi et al., 2014, S. 295, Brenkers/Verboven, 2007, S. 158.

6 Vgl. DoJ/FTC, 2010, S. 2 f., 20 ff.

7 Vgl. DoJ/FTC, 2010, S. $29 \mathrm{ff}$.

8 Vgl. Baker, 2007, S. 3, AAl, 2015, S. 3 f. 
von politischer Einflussnahme agiert. Widerspruch gegen Beschlüsse des Kartellamts ist beim Oberlandgericht Düsseldorf einzulegen. In letzter Instanz entscheidet der Bundesgerichtshof (BGH). Wird ein Zusammenschlussvorhaben auch auf dieser Stufe des Verfahrens untersagt, können die Beteiligten nach § 42 GWB einen Antrag auf Ministererlaubnis stellen. Der Bundesminister für Wirtschaft und Energie kann den Zusammenschluss freigeben, wenn die durch ihn entstehenden Wettbewerbsbeschränkungen von gesamtwirtschaftlichen Vorteilen aufgewogen werden oder ein überragendes Interesse der Allgemeinheit seine Freigabe rechtfertigt.

Die gesetzliche Grundlage zur Beurteilung anmeldungspflichtiger Zusammenschlussvorhaben bildet das Gesetz gegen Wettbewerbsbeschränkungen (GWB). Entscheidendes Kriterium für die Freigabe oder Untersagung ist die Entstehung oder Verstärkung einer marktbeherrschenden Stellung, was der US-amerikanischen Prüfung abgestimmter Verhaltensweisen oder unilateraler Effekte entspricht. ${ }^{9}$ Marktbeherrschung liegt vor, wenn ein Unternehmen auf dem relevanten Markt keinem oder keinem wesentlichen Wettbewerb ausgesetzt ist oder es gegenüber seinen Konkurrenten über eine überragende Marktstellung verfügt ( $\S 18$ Abs. 1 GWB). Entscheidend für die Marktbeherrschungsvermutung sind letztendlich die vom Unternehmen gehaltenen Marktanteile. Darüber hinaus berücksichtigen die Kartellbehörden weitere Faktoren wie die Finanzkraft, den Zugang zu den Beschaffungs- oder Absatzmärkten oder rechtliche oder tatsächliche Schranken für den Marktzutritt anderer Unternehmen.

Zur sachlichen Marktabgrenzung wurde vom Bundeskartellamt lange Zeit im Wesentlichen auf das Bedarfsmarktkonzept abgestellt. ${ }^{10}$ Hiernach sind alle Güter und/oder Dienstleistungen einem Markt zuzuordnen, die aus Verbrauchersicht zur Befriedigung des gleichen Bedürfnisses dienen. ${ }^{11}$ Seit einigen Jahren gelangt jedoch zunehmend der SSNIP-Test zur Anwendung. Ähnlich der US-amerikanischen Efficiency Defense sieht das deutsche Kartellrecht die Möglichkeit der Freigabe wettbewerbsbeschränkender Zusammenschlüsse vor, wenn diese zugleich zur Verbesserung der Wettbewerbsbedingungen führen und diese Verbesserungen die Behinderung des Wettbewerbs überwiegen (§ 36 Abs. 1 S. 2 Nr. 1 GWB).

Medienspezifische Besonderheiten existierten im Rahmen der Zusammenschlusskontrolle bisher lediglich hinsichtlich der Berechnung des Umsatzes der betreffenden Unternehmen. Die

So auch Christiansen, 2000, S. 42.

Vgl. Monopolkommission, 1984, S. 198 Tz. 616.

Vgl. Eine vergleichbare Abgrenzung wird nach der NACE Rev. 2. vorgenommen. Vgl. Eurostat, 2008, S. 305 ff. 
Sonderregeln für den Verlag, die Herstellung und den Vertrieb von Zeitungen und Zeitschriften das Achtfache sowie für die Herstellung, den Vertrieb und die Veranstaltung von Rundfunkprogrammen und den Absatz von Rundfunkwerbezeiten beziehen sich somit ausschließlich auf die Schwellenwerte für die Anmeldepflicht, nicht jedoch auf die Prüfung als solche.

Ebenso wie in den USA war die Würdigung der Besonderheiten von Plattformmärkten bisher gesetzlich nicht vorgeschrieben. Mit den 2017 in Kraft tretenden Änderungen des GWB wird den Kartellbehörden jedoch fortan der Einbezug der Theorie mehrseitiger Marktplätze vorgeschrieben. ${ }^{12}$ Neben den bisherigen Kriterien des $\S 18$ Abs. 3 GWB zu Beurteilung der Marktstellung eines Unternehmens sind fortan u. a. direkte und indirekte Netzwerkeffekte, Multihoming sowie mögliche Größenvorteile im Zusammenhang mit Netzwerkeffekten zu berücksichtigen. ${ }^{13}$

\subsection{Besonderheiten von Medienmärkten}

Die Besonderheit vieler Medienunternehmen gegenüber anderen Unternehmen besteht in der Zusammenführung zweier oder mehrerer Nutzergruppen, die zwar institutionell voneinander getrennt, zugleich jedoch über indirekte Netzeffekte miteinander verbunden sind. ${ }^{14}$ Sie fungieren als Plattform, über welche die verschiedenen Marktseiten koordiniert werden. Der Nutzen der Marktseiten wird nicht alleinig durch den Konsum der Ware oder die Inanspruchnahme der Dienstleistung, sondern ebenso durch die Zahl und/oder die Zusammensetzung der Nutzer auf der anderen Marktseite beeinflusst. ${ }^{15}$ Im Falle positiver Netzeffekte profitieren beide Nutzergruppen von der Existenz der jeweils anderen Marktseite. ${ }^{16}$

Die bestmögliche Ausnutzung der Verbundenheit der Nutzergruppen erfolgt über eine plattformspezifische Preissetzung. ${ }^{17}$ Diese entspricht nicht den für traditionelle Märkten geltenden Regeln, nach denen Unternehmen ihre Preise unter Würdigung der Zahl der konkurrierenden Unternehmen, dem Homogenitätsgrad der Güter, sachlichen, zeitlichen und/oder räumlichen

\footnotetext{
12 Zur wettbewerbsökonomischen Einordnung dieser 9.-ten GWB-Novelle vgl. Budzinski, 2017, Budzinski/Stöhr 2019.

13 Vgl. Deutscher Bundestag, 2016, S. 14.

14 Zu den Besonderheiten von Medienmärkten vgl. grundsätzlich Budzinski/Kuchinke 2018 sowie Dewenter, 2006, S. 60, Dewenter/Haucap, 2009, S. 39.

15 Vgl. Katz/Shapiro, 1985, S. 424, Roson, 2005, S. 144, Schmalensee, 2002, S. 106, Armstrong, 2006, S. 669, Dewenter, 2006, S. 60, Dewenter/Haucap, 2009, S. 39.

16 Vgl. Dewenter/Haucap, 2009, S. 39 f., Wright, 2004, S. 47, Evans, 2010, S. o. A.

17 Vgl. Rochet/Tirole, 2003 S. 990, Schmalensee, 2002, S. 105, Evans/Schmalensee, 2007, S. 154.
} 
Präferenzen sowie der Verteilung der Nachfrager setzen. Vielmehr richten sich die Preise nach der Stärke der indirekten Netzeffekte. Die Gruppe, von welcher der geringere Effekt auf die andere Marktseite ausgeht, zahlt einen höheren Preis als vice versa. Je stärker die Netzeffekte sind, desto geringer ist der von der betreffenden Nutzergruppe zu entrichtende Preis. Mitunter kann dieser weit unter Grenzkosten gesetzt werden oder sogar negative sein. ${ }^{18}$

\subsection{Implikationen für die Zusammenschlusskontrolle}

Die beschriebenen Besonderheiten haben Auswirkungen auf die Beurteilung von Zusammenschlussfällen. Während die durch einen Zusammenschluss entstehende oder verstärkte marktbeherrschende Stellung auf einseitigen Unternehmen infolge der damit einhergehenden Gefahr der missbräuchlichen Nutzung dieser, beispielsweise zur Durchsetzung von Preisen oberhalb des Wettbewerbsniveaus zu Wohlfahrtsverlusten führt, ist auf mehrseitigen Marktplätzen eine differenzierte Betrachtung erforderlich. Da eine monopolistische Plattform besser zur Ausnutzung der indirekten Netzeffekte imstande ist, kann die Gesamtwohlfahrt im Monopol je nach Stärke der indirekten Netzeffekte höher sein als im Wettbewerb. ${ }^{19}$ Bleiben diese Überlegungen im Rahmen der Prüfung der Kartellbehörden unberücksichtigt, könnten Zusammenschlüsse untersagt werden, von denen positive Wohlfahrtseffekte zu erwarten wären. Keinesfalls können Preise oberhalb der Grenzkosten ebenso wenig pauschal als Ausdruck von Marktmacht verstanden werden, wie Preise unterhalb der Grenzkosten durch Verdrängungsabsichten begründet sein müssen. Vielmehr können sie der effizienten Preisstruktur zur bestmöglichen Ausnutzung der indirekten Netzeffekte entsprechen. ${ }^{20}$

Weitere Besonderheiten entstehen bei der Verwendung des SSNIP-Tests zur Marktabgrenzung. Ignoriert die Kartellbehörde die Verbundenheit der Märkte und wendet einen einseitigen SSNIP-Test an, kann es dadurch bereits zu Ungenauigkeiten oder gar Fehlern bei der Marktabgrenzung kommen. Eine mögliche Alternative besteht in der Verwendung der Summe

18 Vgl. Rysman, 2009, S. 130, Wright, 2004, S. 48, Evans/Schmalensee, 2007, S. 160, Evans, 2008, S. 7, Evans, 2003a, S. 193, Dewenter/Rösch/Terschüren, 2014, S. 7.

19 Vgl. Weisman, 2005, S. 137, Klein et al., 2006, S. 595, Dewenter/Haucap/Wenzel, 2011, S. 13 ff. Diese Überlegungen werden aufgegriffen in Lindstädt, 2009, S. 22.

20 Vgl. Schmalensee, 2002, S. 105, Hamelmann/Haucap, 2015, S. 4, Klein et al., 2006, S. 594, Dewenter, 2007, S. 7, Weyl, 2010, S. 1652 f. 
beider Preise. ${ }^{21}$ Hierdurch wäre es möglich, ungeachtet der von gewöhnlichen Märkten abweichenden Preisstruktur eine Einschätzung des Preisniveaus vorzunehmen und Prognosen über die Reaktion der Nachfrage auf Preis(niveau)änderungen abzuleiten. ${ }^{22}$ Weitere Probleme ergeben sich, wenn der Preis auf einem Markt Null ist. Der SSNIP-Test würde ins Leere laufen. ${ }^{23}$ Ebenso ist die alleinige Betrachtung des Markts, auf dem der Preis oberhalb des Wettbewerbspreises liegt, kritisch zu sehen. Werden die Rückkopplungseffekte auf dem verbundenen Markt unberücksichtigt gelassen, fällt der durch den SSNIP-Test prognostizierte Nachfragerückgang c. p. zu gering aus, wodurch der Markt letztendlich zu eng abgegrenzt würde. ${ }^{24}$ Der SSNIP-Test ist somit lediglich unter Anpassungen auf mehrseitige Märkten übertragbar. $^{25}$

Die Ausführungen verdeutlichen die Unmöglichkeit einer pauschalen Bewertung von Zusammenschlussfällen auf mehrseitigen Märkten. ${ }^{26}$ Durch Zusammenschlüsse kann es ebenso zu Effizienzgewinnen wie zu wohlfahrtsmindernden Wettbewerbsbeschränkungen kommen. ${ }^{27}$ Die Beurteilung wird letztendlich vielfach von der konkreten Höhe der indirekten Netzeffekte im Einzelfall abhängen. Hierbei kann es zwar zu Ungenauigkeit mit der Folge von Verzerrungen kommen, ${ }^{28}$ jedoch dürfte den Kartellbehörden in der Regel zumindest die Einschätzung gelingen, von welcher Marktseite der stärkere Netzeffekt ausgeht. ${ }^{29}$ Die Gefahr von Fehlern erster und zweiter Art erscheint somit bei Verzicht auf die Anwendung der Theorie mehrseitiger Märkte höher zu sein als bei Ungenauigkeiten in der Bestimmung der exakten Höhe der Netzeffekte. ${ }^{30}$

\footnotetext{
21 Vgl. Emch/Thompson, 2006, S. 56, Filistrucchi, 2008, S. 15 f., Evans, 2011, S. 149.

22 Vgl. Filistrucchi et al., 2014, S. 331. Kritisch hierzu Kehder, 2013, S. 80 f.

23 Vgl. Auer/Petit, 2015, S. 20.

24 Vgl. Filistrucchi et al., 2014, S. 331, Evans, 2011, S. $165 \mathrm{f}$.

25 Vgl. Evans, 2011, S. 136, Hamelmann/Haucap, 2015, S. 9, Filistrucchi, 2008, S. 17, Filistrucchi et al., 2014, S. 295. Kehder schließt seine Anwendung aufgrund fehlender Anpassungsmöglichkeiten an die Bedingungen auf zweiseitigen Märkten sogar gänzlich aus. Vgl. Kehder, 2013, S. 80 f. Für eine ausführliche Darstellung verschiedener mit der Anwendung des SSNIP-Tests auf zweiseitige Märkte einhergehende Probleme vgl. Dewenter/Rösch/Terschüren, 2014, S. 9 f., Auer/Petit, 2015, S. 20 f.

26 So auch Budzinski/Lindstädt, 2010, S. 440 f., 13, Lindstädt, 2009, S. 41.

27 Vgl. Dewenter/Haucap/Wenzel, 2011, S. 21, Evans/Schmalensee, 2012, S. 34, Budzinski/Lindstädt, 2010, S. 441 f., Evans/Schmalensee, 2007, S. 176.

28 Vgl. Filistrucchi, 2008, S. 10 f. Ähnlich Budzinski/Lindstädt, 2010, S. 442 f.

29 Vgl. Dewenter/Kaiser, 2005, S. 58.

30 Vgl. Dewenter/Rösch/Terschüren, 2014, S. 8 f.
} 
Die Anwendung der Two-Sided Market Theory (TMT) zur Analyse von insbesondere Medienmärkten kann als weithin akzeptiert erachtet werden. Zahlreiche Veröffentlichen legen die Unterschiede zwischen einseitigen und mehrseitigen Marktplätzen und die daraus resultierenden Änderungen in der wettbewerbspolitischen Beurteilung von Plattformen dar. ${ }^{31}$ Im Fokus stehen die Besonderheiten im Rahmen der Prüfung von Missbrauchsvorwürfen und Zusammenschlussfällen. ${ }^{32}$ Während Einigkeit hinsichtlich der Notwendigkeit der kartellrechtlichen Berücksichtigung des Plattformcharakters besteht, ${ }^{33}$ liegen bisher keine Ansätze zur konkreten Umsetzung vor, die über die Forderung einer stärkeren Einzelfallorientierung hinausgehen. Insbesondere die Möglichkeit der Anpassung bestehender Instrumente wie der SSNIPTest wird kontrovers diskutiert. ${ }^{34}$

Empirische Untersuchungen zur Relevanz der TMT für die Entscheidungspraxis der Kartellbehörden beschränken sich auf die inhaltliche Beurteilung einzelner Fälle. Die Ergebnisse lassen weder in der US-amerikanischen noch in der deutschen (und europäischen) Zusammenschlusskontrolle eine stringente Würdigung der Besonderheiten mehrseitiger Märkte erkennen. Wie Evans/Schmalensee (2007) und Auer/Petit (2015) für die USA zeigen, wird die TwoSides Marktes Theorie zwar in einzelnen Fällen angewandt, jedoch erfolgt dies eher episodisch und nicht systematisch. ${ }^{35}$ Kahwaty/Tyler (2014) untersuchen in ihrer Studie kanadische Zusammenschlussfälle und kommen zu dem Schluss einer nicht durchgängigen oder gar nicht erfolgten Würdigung der Theorie mehrseitiger Marktplätze. ${ }^{36}$ Analoge Kritikpunkte bringen Budzinski/Wacker (2007) und Dewenter/Haucap (2009) als Ergebnis ihrer Analyse verschiedener Zusammenschlussvorhaben auf deutschen Medienmärkten vor. Eventuelle Effizienzvorteile durch die jeweiligen Zusammenschlüsse wären nicht hinreichend abgewogen worden. ${ }^{37}$

31 Zu nennen sind hier insbesondere Rochet/Tirole (2003), Evans (2003a), Evans (2003b), Dewenter (2006) und Dewenter/Haucap (2009).

32 Vgl. Dewenter/Haucap (2009), Dewenter/Kaiser (2006), Budzinski/Lindstädt (2010).

33 Vgl. Filistrucchi, 2008, S. 17, White, 2006, S. 8, Filistrucchi et al., 2014, S. 321, Evans/Schmalensee, 2012, S. 11 f., Lindstädt, 2009, S. 33, Dewenter/Rösch/Terschüren, 2014, S. 8, Dewenter/Haucap, 2009, S. 43 f., Evans, 2008, S. 9.

34 Vgl. Evans, 2011, S. 136, Hamelmann/Haucap, 2015, S. 9, Filistrucchi, 2008, S. 17, Filistrucchi et al., 2014, S. 295. Kritsch hierzu vgl. Kehder, 2013, S. 80 f., Dewenter/Rösch/Terschüren, 2014, S. 9 f., Auer/Petit, 2015, S. $20 \mathrm{f}$.

35 Vgl. Evans/Schmalensee, 2007, S. 172 f., Auer/Petit, 2015, S. 22 ff.

36 Vgl. Kahwaty/Tyler, 2014, S. $670 \mathrm{ff}$.

37 Vgl. Budzinski/Wacker, 2007, S. 303, Dewenter/Haucap, 2009, S. 50 ff. 
Eine umfassende und systematische Auswertung des Einbezugs der Theorie in die kartellrechtliche Praxis in den USA und Deutschland fand bisher nicht statt. Diese Forschungslücke soll nachfolgend geschlossen werden.

\section{$4 \quad$ Daten}

\subsection{USA}

In die Untersuchung der praktischen Relevanz der Theorie mehrseitiger Marktplätze wurden alle im Untersuchungszeitraum von 1999 bis Juli 2016 angemeldeten und dem Mediensektor zurechenbaren Zusammenschlussfällen der FTC und der ATD einbezogen, welche über die Websites der Kartellbehörden abrufbar waren. Der Begriff des Medienunternehmens umfasst alle rechtlichen Einheiten, die in den Wertschöpfungsprozess von Medienprodukten involviert sind. Im Sample enthalten sind Unternehmen auf Radio- (6), TV- (11), Kino- (5), Internet- (4), Zeitungs- (2), Software (19) und Mobilfunk- (3) und Werbemärkten (2) sowie Verlage (3). Von den insgesamt betrachteten 55 Fällen wurden 15 von der FTC und 40 von der ATD bearbeitet.

Tabelle 1 gibt einen Überblick über die relevanten Zusammenschlussvorhaben, einschließlich der Kategorisierung als Plattform oder Nicht-Plattform. Nach der Herausnahme aller Fälle, in denen keine Plattform im Sinne der TMT beteiligt war, verblieben 30 zu untersuchende Fälle, die überwiegend von der ATD des DoJ geprüft wurden (93,33 \%). 
Tabelle 1: Übersicht über die ausgewählten Zusammenschlussvorhaben (USA)

\begin{tabular}{|c|c|c|c|c|c|c|c|}
\hline Case Number & Case & Year & Platform & Case Number & Case & Year & Platform \\
\hline 1:99-CV-00993 & $\begin{array}{l}\text { Capstar Broadcasting and } \\
\text { Triathlon Broadcasting }\end{array}$ & 1999 & 1 & 1:08-CV-00746 & $\begin{array}{c}\text { Regal Cinemas, and } \\
\text { Consolidated Theatres } \\
\text { Holdings }\end{array}$ & 2008 & 1 \\
\hline 1:99-CV-01043 & $\begin{array}{c}\text { Citadel, Triathlon, \& Capstar } \\
\text { Broadcasting }\end{array}$ & 1999 & 1 & 0910081 & Dun \& Bradstreet & 2010 & 0 \\
\hline 1:99-CV-01119 & Bell Atlantic & 1999 & 0 & 0910032 & Fidelity National Financial & 2010 & 0 \\
\hline 1:99-CV-00715 & $\begin{array}{l}\text { SBC Communications and } \\
\text { Ameritech }\end{array}$ & 1999 & 0 & 1:10-CV-00846 & $\begin{array}{c}\text { AMC Entertainment } \\
\text { Holdings, and Kerasotes } \\
\text { Showplace Theatres }\end{array}$ & 2010 & 1 \\
\hline 1:99-CV-03212 & CBS & 1999 & 0 & 1:11-CV-00106 & Comcast Corp. & 2011 & 1 \\
\hline 10105 & $\begin{array}{c}\text { America Online, and Time } \\
\text { Warner }\end{array}$ & 2000 & 1 & 1:11-CV-01619 & $\begin{array}{c}\text { Cumulus Media and Citadel } \\
\text { Broadcasting }\end{array}$ & 2011 & 1 \\
\hline 1:00-CV-02789 & $\begin{array}{l}\text { WorldCom, and Intermedia } \\
\text { Communications }\end{array}$ & 2000 & 1 & 1:11-CV-01560 & AT\&T & 2011 & 0 \\
\hline 1:00-CV-02063 & $\begin{array}{c}\text { Clear Channel } \\
\text { Communications and AMFM }\end{array}$ & 2000 & 1 & 1:11-CV-00688 & Google and ITA Software & 2011 & 1 \\
\hline 1:00-CV-01176 & AT\&T and MediaOne Group & 2000 & 0 & 1:11-CV-00948 & H\&R Block & 2011 & 0 \\
\hline kein Angabe & WorldCom and Sprint & 2000 & 0 & 1:12-CV-01354 & Verizon Communications & 2012 & 1 \\
\hline 9910298 & Fidelity National Financial, & 2000 & 0 & 1110172 & $\begin{array}{c}\text { CoStar Group, Lonestar } \\
\text { Acquisition Sub, and } \\
\text { LoopNet }\end{array}$ & 2012 & 1 \\
\hline 0010181 & $\begin{array}{c}\text { Computer Sciences } \\
\text { Corporation, and Mynd }\end{array}$ & 2000 & 0 & 1:13-CV-01984 & Gannett & 2013 & 1 \\
\hline 1:01-CV-01419 & The Thomson Corporation & 2001 & 0 & $\begin{array}{c}\text { 3:13-CV-00133 } \\
\text { WHO }\end{array}$ & Bazaarvoice & 2013 & 1 \\
\hline 1:01-CV-00771 & The News Corporation & 2001 & 1 & 1310159 & $\begin{array}{c}\text { Fidelity National Financial, } \\
\text { and Lender Processing } \\
\text { Services }\end{array}$ & 2013 & 0 \\
\hline 0010077 & MSC.Software Corporation & 2001 & 0 & 1210165 & 2013 Solera Holdings, Inc. & 2013 & 0 \\
\hline 0010212 & $\begin{array}{c}\text { Siemens AG and Vodafone } \\
\text { Group }\end{array}$ & 2001 & 0 & 1:13-CV-00727 & Cinemark Holdings & 2013 & 1 \\
\hline 1:02-CV-02138 & $\begin{array}{c}2 \text { Plaintiff States v. Echostar } \\
\text { Communications }\end{array}$ & 2002 & 1 & 1:14-CV-01186 & $\begin{array}{c}\text { Sinclair Broadcast Group } \\
\text { and Perpetual }\end{array}$ & 2014 & 1 \\
\hline 0210153 & Aspen Technology & 2003 & 0 & 1:14-CV-01823 & $\begin{array}{c}\text { Media General, and LIN } \\
\text { Media }\end{array}$ & 2014 & 1 \\
\hline C 04-0807 VRW & Plaintiff States v. Oracle & 2004 & 0 & 1:14-CV-02007 & Nexstar Broadcasting Group & 2014 & 1 \\
\hline 05-CV-10722 & \begin{tabular}{|c|} 
Plaintiff States v. Marquee \\
Holdings, and LCE Holdings \\
\end{tabular} & 2005 & 1 & $1: 14-C V-8732$ & National CineMedia & 2014 & 1 \\
\hline 1:06-CV-01175 & $\begin{array}{c}\text { The McClatchy Company } \\
\text { and Knight-Ridder }\end{array}$ & 2006 & 1 & 1310199 & CoreLogic & 2014 & 0 \\
\hline 2:07-CV-0329 & $\begin{array}{l}\text { Daily Gazette, and } \\
\text { MediaNews Group } \\
\end{array}$ & 2007 & 1 & 1410085 & Verisk/EagleView & 2014 & 0 \\
\hline 1:08-CV-00899 & Cengage Learning Holdings & 2008 & 0 & $\begin{array}{c}\text { 1:15-CV-02232- } \\
\text { RC } \\
\end{array}$ & $\begin{array}{c}\text { Gray Television, and Schurz } \\
\text { Communications }\end{array}$ & 2015 & 1 \\
\hline 1:08-CV-00143 & $\begin{array}{c}\text { Pearson plc (Reed } \\
\text { Elsevier/Harcourt) }\end{array}$ & 2008 & 0 & 1:15-CV-01119 & $\begin{array}{c}\text { Entercom Communications } \\
\text { and Lincoln Financial Media }\end{array}$ & 2015 & 1 \\
\hline $\begin{array}{l}\text { 1:08-CV01510- } \\
\text { RMU }\end{array}$ & Raycom Media & 2008 & 1 & $1: 15-C V-01583$ & \begin{tabular}{|c} 
Cox Enterprises, Inc., Cox \\
Automotive, and Dealertrack \\
Technologies \\
\end{tabular} & 2015 & 0 \\
\hline 1:08-CV-00245 & Bain Capital & 2008 & 1 & 1:15-CV-02181 & $\begin{array}{c}\text { AMC Entertainment } \\
\text { Holdings, and SMH Theatres }\end{array}$ & 2015 & 1 \\
\hline 0810155 & $\begin{array}{l}\text { CCC Holdings, and Aurora } \\
\text { Equity Partners }\end{array}$ & 2008 & 0 & 1:16-CV-00759 & Charter Communications & 2016 & 1 \\
\hline 0810133 & Reed Elsevier NV & 2008 & 0 & & & & \\
\hline
\end{tabular}

Quelle: Eigene Darstellung. 
Die Analyse der praktischen Bedeutung der Theorie mehrseitiger Märkte beinhaltete in einem ersten Schritt die Betrachtung des zur Marktabgrenzung verwandten Konzepts. Im Fokus standen die Fragen nach der Anpassung des SSNIP-Tests und der Identifikation von Substituten an die plattformspezifischen Besonderheiten. Im nächsten Schritt wurde die Diskussion möglicher negativer Wettbewerbseffekte durch den Zusammenschluss auf das dazu verwandte Instrumentarium untersucht. Relevant waren insbesondere die theoretische Fundierung der Prognose sowie die Vorgehensweise bei der Berechnung der Marktkonzentration sowie bei der Preis- und Umsatzbetrachtung.

\subsection{Deutschland}

Analog zur Vorgehensweise für die USA wurden alle anmeldungspflichtigen Zusammenschlussvorhaben aus den Jahren 1999 bis 2014 auf die Würdigung der Besonderheiten mehrseitiger Marktplätze im Rahmen des Prüfverfahrens systematisch untersucht. Von den insgesamt 40 Fällen des Kartellamts fanden 5 auf Märkten für Bücher statt. Da es sich hierbei nicht um Plattformen im Sinne der Theorie handelt, wurden sie aus der Analyse ausgenommen. Von den verbleibenden 35 Zusammenschlussvorhaben waren in 15 Fällen Märkte für Zeitschriften und in 5 Fällen Märkte für Zeitungen betroffen. Darüber hinaus fanden 3 auf Radiomärkten, 2 auf Internetmärkten und 11 auf TV-Märkten statt. Im Rahmen der Beurteilung dieser 35 Fälle hätte das Kartellamt grundsätzlich auf die Two-Sided Market Theorie zurückgreifen können. Eine Übersicht über die in die Analyse einbezogenen Zusammenschlussvorhaben ist in Tabelle 2 dargestellt: 
Tabelle 2: Übersicht über die ausgewählten Zusammenschlussvorhaben (Deutschland)

\begin{tabular}{|c|c|c|c|c|c|c|c|}
\hline Case Number & Case & Year & Platform & Case Number & Case & Year & Platform \\
\hline B6-145-98 & Rheinpfalz/AZ & 1999 & yes & B6-086-05 & MSV/PVN/Buch\&Presse & 2005 & yes \\
\hline B6-020-00 & Holtzbrinck/Südkurier & 2000 & yes & B6-103-04 & S-W/Wochenspiegel & 2005 & yes \\
\hline B6-088-00 & AS/yeshr & 2000 & yes & B6-106-04 & Volksfreund/TW & 2005 & yes \\
\hline B6-118-98 & WAZ/OTZ & 2000 & yes & B7-022-05 & Iesy/Ish & 2005 & yes \\
\hline B6-056-01 & SV-C/WEKA & 2001 & yes & B7-038-05 & BC Partners/Ish & 2005 & yes \\
\hline B7-205-00 & CNRW/NetCologne & 2001 & yes & B6-103-05 & AS/P7S1 & 2006 & yes \\
\hline B6-033-02 & WAZ/Schachenmeyer & 2002 & yes & B6-138-05 & SV/Lokalzeitung & 2006 & yes \\
\hline B6-098-02 & Holtzbrinck/G+J & 2002 & yes & B6-142-05 & RTL/n-tv & 2006 & yes \\
\hline B6-119-02 & RTL/AVE & 2002 & yes & B6-008-08 & Thalia/BuchKaiser & 2008 & no \\
\hline B6-136-01 & G+J/berlin.de & 2002 & yes & B6-052-08 & Intermedia/HB & 2008 & yes \\
\hline B6-144-01 & T-Online/AS & 2002 & yes & B7-200-07 & KDG/Orion & 2008 & yes \\
\hline B6-159-01 & Radio L 12 & 2002 & yes & B6-009-09 & Bertelsmann/Brockhaus & 2009 & no \\
\hline B7-168-01 & Liberty Media/DT & 2002 & yes & B6-038-09 & mhn/AS & 2009 & yes \\
\hline B6-007-03 & Bertelsmann/Heyne & 2003 & no & B6-150-08 & NPG/Schwäbisch Hall & 2009 & yes \\
\hline B6-026-04 & G+J/RBA & 2004 & yes & B6-098-09 & Roth+Horsch/PV Pfalz & 2010 & no \\
\hline B6-027-04 & DuMont/Bonner Druckerei & 2004 & yes & B6-094-10 & P7S1/RTL & 2011 & yes \\
\hline B6-056-03 & Radio TON/Lokalradio & 2004 & yes & B7-066-11 & LGI/KBW & 2011 & yes \\
\hline B6-081-03 & KG WK/LR MV & 2004 & yes & B6-084-11 & Libri/Könemann & 2012 & no \\
\hline B6-120-03 & Holtzbrinck/G+J II & 2004 & yes & B7-070-12 & KDG/Tele Columbus & 2013 & yes \\
\hline B7-150-04 & SES/Premiere & 2004 & yes & B6-098-13 & Funke/AS & 2014 & yes \\
\hline
\end{tabular}

Quelle: Eigene Darstellung.

Die systematische Untersuchung der Beschlüsse umfasste sowohl die Marktabgrenzung als auch die Entstehung oder Verstärkung einer marktbeherrschenden Stellung. Auf der Ebene der Marktabgrenzung lag der Fokus wiederum auf der Frage, anhand welches Konzepts die Definition des relevanten Marktes vorgenommen wird und inwiefern die Mehrseitigkeit von Plattformen dabei Berücksichtigung erfahren hat. Hinsichtlich der Marktbeherrschung wurde untersucht, auf welcher theoretischen Grundlage die Prognose eventueller Marktmachtsprobleme erfolgte, der Anzeigen-Auflagen-Spirale oder der Theorie mehrseitiger Marktplätze, und ob Preise und Umsätze nach den Regeln einseitiger Märkte oder plattformspezifisch betrachtet wurden.

\section{$5 \quad$ Analyse}

\subsection{USA}

\subsubsection{Marktabgrenzung}

Die Ergebnisse der systematischen Untersuchung der relevanten Zusammenschlussfälle im Untersuchungszeitraum sind, untergliedert nach der Stufe der Marktabgrenzung und der sich anschließenden Prognose der Wettbewerbswirkungen, in nachstehender Tabelle 3 zusammengefasst: 
Tabelle 3: Gesamtübersicht über die TMT in der Zusammenschlussprüfung (USA)

\begin{tabular}{|c|c|c|c|c|c|c|c|c|c|c|}
\hline \multirow{2}{*}{ Case } & \multirow{2}{*}{ Jahr } & \multicolumn{3}{|c|}{ Market Definition } & \multicolumn{6}{|c|}{ SIEC } \\
\hline & & SNNIP & Substitutes & TMT & Prices & TMT & Revenues & TMT & Theory & HHI \\
\hline $\begin{array}{c}\text { Capstar Broadcasting and } \\
\text { Triathlon Broadcasting } \\
\end{array}$ & 1999 & yes & yes & no & yes & no & yes & no & none & yes \\
\hline $\begin{array}{c}\text { Citadel, Triathlon, \& Capstar } \\
\text { Broadcasting } \\
\end{array}$ & 1999 & yes & yes & no & yes & no & no & no & none & yes \\
\hline America Online, and Time Warner & 2000 & no & no & - & no & - & no & - & none & no \\
\hline $\begin{array}{c}\text { WorldCom, and Intermedia } \\
\text { Communications } \\
\end{array}$ & 2000 & yes & no & no & yes & no & yes & no & none & no \\
\hline $\begin{array}{c}\text { Clear Channel Communications } \\
\text { and AMFM }\end{array}$ & 2000 & yes & yes & no & yes & no & yes & no & none & yes \\
\hline The News Corporation & 2001 & yes & no & no & yes & no & no & - & none & yes \\
\hline $\begin{array}{l}2 \text { Plaintiff States v. Echostar } \\
\text { Communications }\end{array}$ & 2002 & yes & yes & no & yes & no & yes & no & none & yes \\
\hline $\begin{array}{c}\text { Plaintiff States v. Marquee } \\
\text { Holdings, and LCE Holdings }\end{array}$ & 2005 & yes & yes & no & yes & no & yes & no & none & yes \\
\hline $\begin{array}{l}\text { The McClatchy Company and } \\
\text { Knight-Ridder }\end{array}$ & 2006 & yes & yes & yes & yes & yes & yes & yes & yes & yes \\
\hline $\begin{array}{c}\text { Daily Gazette, and MediaNews } \\
\text { Group }\end{array}$ & 2007 & yes & yes & yes & yes & yes & yes & yes & yes & no \\
\hline Raycom Media & 2008 & yes & no & no & yes & no & yes & no & none & yes \\
\hline Bain Capital & 2008 & yes & yes & no & yes & no & no & - & none & yes \\
\hline $\begin{array}{c}\text { Regal Cinemas, and Consolidated } \\
\text { Theatres Holdings }\end{array}$ & 2008 & yes & yes & no & yes & no & yes & no & none & yes \\
\hline $\begin{array}{c}\text { AMC Entertainment Holdings, and } \\
\text { Kerasotes Showplace Theatres }\end{array}$ & 2010 & no & yes & - & yes & no & yes & no & none & yes \\
\hline Comcast Corp. & 2011 & yes & no & no & yes & no & yes & no & none & no \\
\hline $\begin{array}{l}\text { Cumulus Media and Citadel } \\
\text { Broadcasting } \\
\end{array}$ & 2011 & yes & yes & no & yes & no & yes & no & none & yes \\
\hline Google and ITA Software & 2011 & yes & yes & no & no & no & yes & no & none & no \\
\hline Verizon Communications & 2012 & yes & no & no & yes & no & no & - & none & no \\
\hline $\begin{array}{c}\text { CoStar Group, Lonestar } \\
\text { Acquisition Sub, and LoopNet }\end{array}$ & 2012 & no & no & - & no & - & no & - & none & no \\
\hline Gannett & 2013 & yes & no & no & yes & no & yes & no & none & yes \\
\hline Bazaarvoice & 2013 & no & no & - & yes & no & yes & no & none & yes \\
\hline Cinemark Holdings & 2013 & yes & yes & no & yes & no & yes & no & none & yes \\
\hline $\begin{array}{c}\text { Sinclair Broadcast Group and } \\
\text { Perpetual }\end{array}$ & 2014 & yes & yes & no & yes & no & yes & no & none & yes \\
\hline Media General, and LIN Media & 2014 & yes & yes & no & yes & no & yes & no & none & yes \\
\hline Nexstar Broadcasting Group & 2014 & yes & yes & no & yes & no & yes & no & none & yes \\
\hline National CineMedia & 2014 & yes & yes & no & yes & no & yes & no & none & yes \\
\hline $\begin{array}{l}\text { Gray Television, and Schurz } \\
\text { Communications }\end{array}$ & 2015 & yes & yes & no & yes & no & yes & no & none & yes \\
\hline $\begin{array}{l}\text { Entercom Communications and } \\
\text { Lincoln Financial Media }\end{array}$ & 2015 & yes & yes & no & yes & no & yes & no & none & yes \\
\hline $\begin{array}{l}\text { AMC Entertainment Holdings, and } \\
\text { SMH Theatres }\end{array}$ & 2015 & yes & yes & no & yes & no & no & - & none & yes \\
\hline Charter Communications & 2016 & yes & no & no & yes & no & yes & no & none & no \\
\hline
\end{tabular}

Quelle: Eigene Darstellung.

Entgegen der auf theoretischer Ebene bestehenden Anwendungsprobleme des SSNIP-Tests bei Plattformen griffen die US-amerikanischen Kartellbehörden in 26 der 30 betrachteten Fälle $(86,67 \%)$ auf diesen zurück. Eine Anpassung der klassischen Vorgehensweise bei der Verwendung des hypothetischen Monopolistentests erfolgte lediglich in zwei der 26 (7,69 \%) Fälle in den Jahren 2006 und 2007. In allen anderen wurde eine Marktabgrenzung wie auf einseitigen Märkten vorgenommen. Grundsätzlich besteht somit die Gefahr der fehlerhaften Ermittlung 
von Preisreaktionen. Die mit dem deutschen Bedarfsmarktkonzept vergleichbare Betrachtung von Substitutionsbeziehungen fand in 20 von 30 Fällen (66,67 \%) statt, jedoch wiederum ohne plattformspezifischen Charakteristika Rechnung zu tragen. Ungeachtet der Besonderheiten mehrseitiger Märkte gelangt noch immer das klassische tradierte Instrumentarium zum Einsatz. Zudem lässt die Betrachtung der Jahreszahlen keinen Trend zur stärkeren Würdigung im Zeitablauf erkennen. Seit den beiden Fällen in 2006 und 2007 wurde die Theorie mehrseitiger Märkte nicht mehr thematisiert. Für die Abgrenzung des relevanten Markts hat sie seither nicht an praktischer Relevanz gewonnen.

\subsubsection{Marktbeherrschung}

Gleiches Bild stellt sich für die Prognose einer signifikanten Reduktion des Wettbewerbs dar. Weder bei der Berechnung der Umsätze noch der Preise wurden plattformspezifische Abweichungen von der klassischen ökonomischen Theorie in die Analyse einbezogen. Die in der deutschen Kartellrechtspraxis lange Zeit gebräuchliche Anzeigen-Auflagen-Spirale besitzt für die US-amerikanische Fusionskontrolle keine Relevanz. Jedoch hat auch die Two-Sided Markets Theory mit ihrer zunehmenden wissenschaftlichen Anerkennung keinen Einzug in die Prüfung von anmeldungspflichtigen Zusammenschlussvorhaben gefunden. Lediglich in den beiden Zusammenschlussfällen, in denen eine Marktabgrenzung nach den Vorgaben der TMT vorgenommen wurde, griffen die Kartellbehörden bei der Prognose potenzieller Wettbewerbswirkungen auf die theoretischen Überlegungen der Plattformenökonomie zurück. In 25 der 27 Fälle, bei denen Preisbetrachtungen angestrebt wurden, fanden die Bewertungsregeln einseitiger Märkte Anwendung. Eine plattformspezifische Diskussion der Preise beschränkte sich demnach auf 7,40 \% der relevanten Fälle. Lediglich in diesen beiden Fällen erfolgte zudem ein Einbezug der TMT in die Analyse der Umsätze, wohingegen in weiteren 18 Zusammenschlussprüfungen eine herkömmliche Umsatzbetrachtung stattfand.

Wie aus Tabelle 3 weiterhin hervorgeht, wird (der Veränderung) der Marktkonzentration eine hohe Relevanz bei der Beurteilung von Zusammenschlüssen beigemessen. In 22 der 30 Fälle (73,33 \%) wurde der HHI berechnet, allerdings erneut ohne Berücksichtigung der Verbundenheit der Märkte. 
Ebenso wie bei der sachlichen Marktabgrenzung ist keine im Zeitablauf zunehmende Integration der Theorie mehrseitiger Marktplätze in die Prognose der Wettbewerbswirkungen geplanter Zusammenschlussvorhaben ersichtlich.

\subsection{Deutschland}

\subsubsection{Marktabgrenzung}

Bei der Betrachtung der theoretischen Fundierung der Marktabgrenzung wird zunächst die unterschiedliche Vorgehensweise der deutschen und der US-amerikanischen Kartellbehörden deutlich. Der in den USA mehrheitlich verwandte SSNIP-Test weist in Deutschland bisher eine geringe praktische Relevanz zur Ermittlung des relevanten Markts auf. Lediglich in vier der 34 untersuchten Fälle wandte das Bundeskartellamt ihn in Ergänzung zum Bedarfsmarktkonzept an, welches ungeachtet der zunehmenden Europäisierung des deutschen Kartellrechts noch immer das Standardinstrument zur Marktabgrenzung darstellt. In neun Fällen wurde dies durch die Betrachtung der Angebotsflexibilität ergänzt.

Wie aus Tabelle 4 hervorgeht, nahmen die Kartellbehörden im Großteil der Fälle eine einseitige Marktabgrenzung vor. Alleinig in den Zusammenschlussvorhaben von Intermedia/HB (2008), KDG/Orion (2008) und KDG/Tele Columbus (2011) wurde die Existenz interdependenter Nutzergruppen bei der Ermittlung des relevanten Markts berücksichtigt. Den Besonderheiten von Medienmärkten wurde somit lediglich in 3 von 35 Fällen (8,57 \%) Rechnung getragen. In allen anderen Fällen erfolgte ungeachtet des Vorliegens (mindestens) einer Plattform eine einseitige Abgrenzung wie auf traditionellen Märkten. Unerheblich war dabei, welches theoretische Konzept als Grundlage der Marktabgrenzung zur Anwendung gelangte. Die unter Punkt 2.3 beschriebenen Probleme bei der Anwendung des SSNIP-Tests auf mehrseitige Märkte lassen sich daher nicht (alleinig) als Ursache für die bisher unzulängliche Integration der TMT auf dieser Ebene der Prüfung anführen. Die zunehmende wissenschaftliche Anerkennung der Theorie im Verlauf des Untersuchungszeitraums spiegelt sich auf dieser Ebene der kartellrechtlichen Praxis nicht wider. Ein nennenswerter Unterschied zur Vorgehensweise der FTC und der ATD des DoJ ist nicht ersichtlich. 
Tabelle 4: Gesamtübersicht über die TMT in der Zusammenschlussprüfung

\begin{tabular}{|c|c|c|c|c|c|c|c|c|}
\hline \multirow{2}{*}{ Case } & \multirow{2}{*}{ Year } & \multicolumn{2}{|c|}{ Market Definition } & \multicolumn{5}{|c|}{ Market Concentration } \\
\hline & & Concept & TMT & Prices & TMT & Revenues & TMT & Theory \\
\hline Rheinpfalz/AZ & 1999 & demand-side oriented & no & no & - & no & - & none \\
\hline Holtzbrinck/Südkurier & 2000 & demand-side oriented & no & yes & no & no & - & none \\
\hline AS/yeshr & 2000 & $\begin{array}{l}\text { demand-side oriented, } \\
\text { supply-side flexibility }\end{array}$ & no & no & - & no & - & none \\
\hline WAZ/OTZ & 2000 & demand-side oriented & no & no & - & yes & no & none \\
\hline SV-C/WEKA & 2001 & $\begin{array}{l}\text { demand-side oriented, } \\
\text { supply-side flexibility }\end{array}$ & no & no & - & yes & yes & none \\
\hline CNRW/NetCologne & 2001 & demand-side oriented & no & no & - & no & - & none \\
\hline WAZ/Schachenmeyer & 2002 & demand-side oriented & no & yes & yes & no & - & $\begin{array}{c}\text { advertising } \\
\text { circulations } \\
\text { piral }\end{array}$ \\
\hline Holtzbrinck/G+J & 2002 & demand-side oriented & no & yes & no & yes & yes & $\begin{array}{c}\text { advertising } \\
\text { circulations } \\
\text { piral }\end{array}$ \\
\hline RTL/AVE & 2002 & demand-side oriented & no & no & - & yes & no & none \\
\hline $\mathrm{G}+\mathrm{J} /$ berlin.de & 2002 & demand-side oriented & no & no & - & no & - & none \\
\hline T-Online/AS & 2002 & demand-side oriented & no & no & - & no & - & none \\
\hline Radio L 12 & 2002 & demand-side oriented & no & no & - & no & - & none \\
\hline Liberty Media/DT & 2002 & $\begin{array}{l}\text { demand-side oriented, } \\
\text { supply-side flexibility }\end{array}$ & no & yes & yes & yes & yes & none \\
\hline $\mathrm{G}+\mathrm{J} / \mathrm{RBA}$ & 2004 & $\begin{array}{l}\text { demand-side oriented, } \\
\text { supply-side flexibility }\end{array}$ & no & no & - & no & - & none \\
\hline $\begin{array}{l}\text { DuMont/Bonner } \\
\text { Druckerei }\end{array}$ & 2004 & demand-side oriented & no & no & - & yes & no & $\begin{array}{c}\text { advertising } \\
\text { circulations } \\
\text { piral }\end{array}$ \\
\hline Radio TON/Lokalradio & 2004 & demand-side oriented & no & yes & no & yes & no & none \\
\hline KG WK/LR MV & 2004 & demand-side oriented & no & no & - & yes & no & $\begin{array}{c}\text { advertising } \\
\text { circulations } \\
\text { piral }\end{array}$ \\
\hline Holtzbrinck/G+J II & 2004 & demand-side oriented & no & yes & no & yes & no & \begin{tabular}{|c} 
advertising \\
circulations \\
piral
\end{tabular} \\
\hline SES/Premiere & 2004 & $\begin{array}{l}\text { demand-side oriented, } \\
\text { supply-side flexibility }\end{array}$ & no & no & - & no & - & none \\
\hline MSV/PVN/Buch\&Presse & 2005 & demand-side oriented & no & no & - & no & - & $\begin{array}{c}\text { advertising } \\
\text { circulations } \\
\text { piral }\end{array}$ \\
\hline S-W/Wochenspiegel & 2005 & demand-side oriented & no & no & - & yes & no & $\begin{array}{c}\text { advertising } \\
\text { circulations } \\
\text { piral }\end{array}$ \\
\hline Volksfreund/TW & 2005 & demand-side oriented & no & no & - & yes & no & $\begin{array}{c}\text { advertising } \\
\text { circulations } \\
\text { piral }\end{array}$ \\
\hline Iesy/Ish & 2005 & $\begin{array}{l}\text { demand-side oriented, } \\
\text { supply-side flexibility, } \\
\text { SSNIP-Test }\end{array}$ & no & yes & yes & no & - & none \\
\hline BC Partners/Ish & 2005 & $\begin{array}{l}\text { demand-side oriented, } \\
\text { supply-side flexibility, } \\
\text { SSNIP-Test }\end{array}$ & no & yes & yes & no & - & none \\
\hline AS/P7S1 & 2006 & demand-side oriented & no & yes & no & yes & no & none \\
\hline SV/Lokalzeitung & 2006 & demand-side oriented & no & no & - & yes & yes & $\begin{array}{c}\text { advertising } \\
\text { circulations } \\
\text { piral }\end{array}$ \\
\hline RTL/n-tv & 2006 & demand-side oriented & no & yes & no & yes & no & none \\
\hline Intermedia/HB & 2008 & $\begin{array}{l}\text { demand-side oriented, } \\
\text { supply-side flexibility }\end{array}$ & yes & yes & yes & yes & yes & TMT \\
\hline KDG/Orion & 2008 & $\begin{array}{l}\text { demand-side oriented, } \\
\text { supply-side flexibility }\end{array}$ & yes & yes & no & yes & no & none \\
\hline $\mathrm{mhn} / \mathrm{AS}$ & 2009 & demand-side oriented & no & no & - & no & - & TMT \\
\hline NPG/Schwäbisch Hall & 2009 & $\begin{array}{c}\text { demand-side oriented, } \\
\text { supply-side flexibility, } \\
\text { SSNIP-Test }\end{array}$ & no & yes & no & yes & yes & TMT \\
\hline P7S1/RTL & 2011 & demand-side oriented & no & yes & no & yes & no & none \\
\hline LGI/KBW & 2011 & $\begin{array}{l}\text { demand-side oriented, } \\
\text { supply-side flexibility }\end{array}$ & yes & yes & yes & yes & yes & TMT \\
\hline KDG/Tele Columbus & 2013 & $\begin{array}{l}\text { demand-side oriented, } \\
\text { supply-side flexibility }\end{array}$ & no & yes & yes & yes & yes & TMT \\
\hline Funke/AS & 2014 & $\begin{array}{c}\text { demand-side oriented, } \\
\text { supply-side flexibility, } \\
\text { SSNIP-Test }\end{array}$ & no & yes & yes & yes & yes & TMT \\
\hline
\end{tabular}

Quelle: Eigene Darstellung. 


\subsubsection{Marktbeherrschung}

Die US-amerikanischen Daten zeigen eine geringere praktische Relevanz der TMT sowohl auf der Ebene der Marktabgrenzung als auch bei der Beurteilung der Gefahr der Monopolisierung. Während die Vorgehensweise der deutschen Kartellbehörden bei der Definition des relevanten Markts vergleichbare diesbezügliche Defizite aufweist, ist eine im Zeitablauf zunehmende Würdigung der Besonderheiten von Plattformen bei der Prüfung der Entstehung oder Verstärkung einer marktbeherrschenden Stellung zu erkennen.

Lange Zeit wurde die Verbundenheit von Anzeigen- und Lesermärkten anhand der sog. Anzeigen-Auflagen-Spirale dargestellt. Der Einfluss der einen Seite auf die andere Seite läuft nicht allein über die Größe der jeweiligen Nutzergruppe, sondern über die Qualität. Steigt die Nachfrage auf dem Lesermarkt, wird eine Zeitung interessanter für Werbekunden. Die Werbeeinnahmen steigen c. p. und werden zur Verbesserung der redaktionellen Inhalte verwendet, wodurch sich die Qualität der Zeitung und damit wiederum die Nachfrage auf dem Lesermarkt erhöhen. ${ }^{38}$ Die Annahme einer steten Qualitätsverbesserung einer Zeitung bei steigender Nachfrage auf dem Leser- und Werbemarkt wurde jedoch nach und nach kritisiert. ${ }^{39}$ Der Umweg über die Qualität entfällt bei der Theorie mehrseitiger Marktplätze, welche die Wechselwirkungen zwischen beiden Marktseiten über die indirekten Netzeffekte erklärt. Die in der wissenschaftlichen Literatur erfolgte Abkehr von der Anzeigen-Auflagen-Spirale spiegelt sich in der theoretischen Fundierung der Marktmachtsdiskussion der deutschen Kartellbehörden wider. Diente bis zum Jahre 2007 noch ausschließlich die Anzeigen-Auflagen-Spirale als theoretische Grundlage, wurde diese seither gänzlich durch die Two-Sided Markets Theory ersetzt. Weniger stringent fällt die Würdigung der plattformspezifischen Preisstruktur aus. In 17 der insgesamt zu prüfenden 35 Zusammenschlussvorhaben nahm das Kartellamt eine Preisbetrachtung vor. Zwar trugen sie in 8 dieser Fälle $(47,06 \%)$ der von einseitigen Märkten abweichenden Preisstruktur Rechnung, eine im Zeitablauf zunehmender und systematischer werdender Einbezug ist allerdings nicht ersichtlich. Auch in den 7 Fällen seit 2008 erfolgte die Analyse der relevanten Preise nach den Regeln einseitiger Märkte. Die Umsatzbetrachtung lässt keine häufigere Anwendung der TMT erkennen als die der Preise. Von den 21 der insgesamt 35 Zusammenschlussprüfungen führten die Kartellbehörden lediglich 9 (42,86 \%) unter

\footnotetext{
38 Vgl. Nussberger, 1961, S. 16, Gustafsson, 1978, S.1 ff.

39 Vgl. hierzu und zu weiterer Kritik an der Anzeigen-Auflagen-Spirale exemplarisch Hass, 2007, S. 73.
} 
Berücksichtigung von Netzeffekten und der Verbundenheit der Märkte durch. Sowohl Preise als auch Umsätze wurden in 14 der 35 Fälle in die Analyse einbezogen. Die diesbezüglichen plattformspezifischen Besonderheiten wurden dabei jedoch lediglich in 4 dieser 14 (28,57 \%) Prüfungen diskutiert.

Zusammenfassend kann der Einbezug der Theorie mehrseitiger Marktplätze in die Prüfung des Kartellamts bisher nicht als konsequent und stringent bezeichnet werden. Vielmehr handelte es sich um eine vereinzelte Würdigung der Verbundenheit von Märkten über indirekte Netzeffekte. Häufig greifen die Kartellbehörden noch immer auf ihr tradiertes Instrumentarium zurück, ohne dies an die Besonderheiten von Medienunternehmen anzupassen. Grundsätzlich birgt dieses Verhalten die Gefahr einer fehlerhaften Einschätzung der Entwicklung von Preisen und Mengen nach dem Zusammenschluss, indem beispielsweise der Preissetzungsspielraum der Plattform durch die Vernachlässigung der Disziplinierungswirkung der Netzeffekte überschätzt wird.

\subsection{Vergleichende Gesamtbetrachtung}

Die zusammenfassenden Ergebnisse für die Marktabgrenzung und die Prognose der Gefahr der Monopolisierung in der US-amerikanischen Fusions- und einer marktbeherrschenden Stellung in der deutschen Zusammenschlusskontrolle in den Abbildungen 3 und 4 verdeutlichen die bisher unzulängliche Einbindung der Two-Sided Market Theory in das Prüfverfahren der Kartellbehörden. Zwar erscheint die Würdigung der Besonderheiten von Plattformen im deutschen Kartellrecht auf den ersten Blick zumindest im Bereich der Marktmachtsdiskussion deutlich ausgeprägter zu sein als in den USA, als hier seit dem Jahr 2008 eine Tendenz zur stärkeren Integration der Mehrseitigkeit von Marktplätzen zu beobachten ist. Zumindest in zirka der Hälfte der Fälle erfolgte eine plattformspezifische Preis- und Umsatzbetrachtung. Der anfängliche Befund einer umfassenderen Integration der TMT durch die deutschen als durch die US-amerikanischen Kartellbehörden wird durch die Gesamtbetrachtung der empirischen Ergebnisse jedoch zu einem gewissen Grad relativiert. Während FTC und DoJ bisher weitgehend auf den Einbezug verzichtet haben, erfolgt dieser in Deutschland bisher wenig systematisch. Unter allen 12 Zusammenschlussfallprüfungen seit 2008 waren lediglich zwei, die durchgängig im Sinne der TMT geprüft wurden (Intermedia/HB und LGI/KBW). Bei zwei 
weiteren Fällen (KDG/Tele Columbus und Funke/Axel-Springer) verzichteten die Kartellbehörden zwar auf eine mehrseitige Marktabgrenzung, würdigten die von einseitigen Märkten abweichenden Verhaltensweisen von Plattformen jedoch im Rahmen der Preis- und Umsatzbetrachtung. Im Falle von KDG/Orion wurde die Wirkung von Netzeffekten zwar in die Definition des relevanten Markts, nicht jedoch in die Preis- und Umsatzbetrachtung einbezogen. In 83,33 \% der seit 2008 geprüften Fälle wurden die Besonderheiten von Medienmärkten demgemäß gar nicht oder nur sporadisch gewürdigt.

Wie unter Punkt 2.3 ausgeführt, kann es aus theoretischer Sicht infolge der fehlenden Betrachtung der Besonderheiten mehrseitiger Marktplätze zu Fehlentscheidungen kommen. Insbesondere besteht die Gefahr der Überschätzung der negativen Wohlfahrtswirkungen bei zeitgleicher Unterschätzung möglicher Effizienzgewinne. Es läge somit die Vermutung nahe, bei fehlender Würdigung von Netzeffekten und deren Wirkung seien Zusammenschlussvorhaben untersagt worden, die durchaus positive Wohlfahrtseffekte haben hätten können. Eine abschließende Beurteilung möglicher Auswirkungen der unsystematischen oder gar fehlenden Berücksichtigung kann ohne eine konkrete Einzelfallanalyse nicht beantwortet werden. Hinweise hierauf lassen die vorliegenden Daten weder für die USA noch für Deutschland erkennen.

Tabelle 5 gibt einen Überblick über die Anzahl angemeldeter Zusammenschlüsse von Plattformen und Nicht-Plattformen, die im Untersuchungszeitraum durch FTC und ATD in den USA und das Bundeskartellamt in Deutschland ohne Auflagen oder mit Auflagen freigegeben oder untersagt wurden.

Tabelle 5: Urteile (USA und Deutschland)

\begin{tabular}{|c|c|c|c|c|}
\hline & \multicolumn{2}{|c|}{ USA } & \multicolumn{2}{c|}{ Germany } \\
\hline Decision & Platform & No Platform & Platform & No Platform \\
\hline Clearance & 0 & 1 & 15 & 4 \\
\hline Conditional Clearance & 28 & 19 & 4 & - \\
\hline Prohibition & 2 & 5 & 17 & - \\
\hline
\end{tabular}

Quelle: Eigene Darstellung.

Werden zunächst lediglich die US-amerikanischen Fälle betrachtet, treten keine nennenswerten Unterschiede hervor. Obgleich die Kartellbehörden den plattformspezifischen Besonderheiten im Rahmen ihrer Prüfung in der Mehrheit der Fäll keinerlei Bedeutung beigemessen 
haben, wurden Zusammenschlüsse auf mehrseitigen Märkten nicht häufiger untersagt oder lediglich unter Auflagen freigegeben als auf herkömmlichen Märkten. Während sich in den USA eine vergleichsweise lockere Kartellpolitik mit häufigen Freigaben unter Auflagen erkennen lässt, erscheint die deutsche Zusammenschlusskontrolle bei dieser groben Betrachtung der Daten restriktiver zu sein. Mit 17 der untersuchten 36 Fälle auf Medienmärkten wurden 47,22 \% aller angemeldeten Zusammenschlussvorhaben untersagt. Im Gegenzug kommen Auflagen eine wesentlich geringere Bedeutung zu als in den USA. Wird eine Freigabe erteilt, erfolgt dies selbst unter Beteiligung von Plattformen in der Regel ohne Nebenbedingungen. Wird der Fokus ausschließlich auf die 7 Zusammenschlussfälle seit dem Jahr 2008 gelegt, bei denen die Theorie mehrseitiger Marktplätze keine Würdigung fand, wurden davon 5 ohne Nebenbedingungen freigegeben, zwei untersagt. Bei den 5 Fällen mit teilweiser oder konsequenter Anwendung der Theorie kam es zu einer Freigabe ohne Nebenbedingungen, zwei mit Nebenbedingungen und zwei Untersagungen. Eine tendenziell häufigere Freigabe von Zusammenschlussvorhaben (mit oder ohne Auflagen) bei Berücksichtigung der Zweiseitigkeit von Märkten ist insofern nicht ersichtlich.

Auch die Betrachtung der Art der Auflagen für Plattformen und Nicht-Plattformen in den USA lässt keine strukturellen Unterschiede erkennen.

Tabelle 6: Auflagen (USA und Deutschland)

\begin{tabular}{|c|c|c|c|c|}
\hline & \multicolumn{2}{|c|}{ USA } & \multicolumn{2}{c|}{ Germany } \\
\hline Conditions & Platform & No Platform & Platform & No Platform \\
\hline Divestitures & 21 & 16 & - & - \\
\hline Conduct & 6 & 1 & - & - \\
\hline Divestitures \& Conduct & 1 & 1 & 4 & - \\
\hline Restructuring & - & 1 & - & \\
\hline
\end{tabular}

Quelle: Eigene Darstellung.

Wie aus Tabelle 6 hervorgeht, wurden beiden Gruppen in vergleichbarem Ausmaß überwiegend Verkäufe auferlegt und gelegentlich bestimmte Verhaltensweisen vorgegeben oder untersagt. Inwiefern die Würdigung der Theorie mehrseitiger Marktplätze zu einer häufigeren Freigabe ohne Auflagen oder geringeren Auflagen geführt hätte, kann an dieser Stelle ohne 
tiefergehende Analyse der Fälle nicht beantwortet werden. Vermuten ließen sich bei konsequenter Anwendung der TMT geringere Vorgaben zum Marktverhalten und/oder Verkäufen von Anteilen als bei Nicht-Plattformen.

Zusammenfassend kann weder für die US-amerikanische noch für die deutsche Zusammenschlusskontrolle eine hohe Relevanz der TMT für die kartellrechtliche Praxis konstatiert werden. Zwar trägt das Bundeskartellamt den plattformspezifischen Besonderheiten in ihren Ausführungen seit 2008 häufiger Rechnung als FTC und ATD, eine konsequente und systematische Anwendung erfolgt bisher jedoch auch hier nicht. Die Gefahr von Fehlentscheidungen ist somit grundsätzlich gegeben. Zu welchem Grad die beschriebenen Schwierigkeiten bei der konkreten Umsetzung der theoretischen Erkenntnisse auf das Prüfverfahren ausschlaggebend hierfür sind, kann durchaus kritisch diskutiert werden.

\subsection{Kritische Reflexion}

Aus der vorstehenden Analyse gehen die Defizite bei der Integration der Erkenntnisse der Plattformökonomie in die Prüfung der Kartellbehörden deutlich hervor. Die vom deutschen Gesetzgeber angestrebte Aufnahme in das Gesetz gegen Wettbewerbsbeschränkungen erscheint vor diesem Hintergrund durchaus sinnvoll. Das Bundeskartellamt selbst begrüßt die Änderungen. Zwar hätte es die neuen wissenschaftlichen Erkenntnisse in seiner bisherigen Praxis bereits versucht, in seine Entscheidungen aufzunehmen „[...] soweit sie für die kartellbehördliche Praxis anwendbar waren $[. . .]^{140}$, eine gesetzliche Verankerung sei dennoch ratsam.

Etwaige Bestrebungen lassen sich in den USA derzeit nicht erkennen. Die offenbar unterschiedliche Gewichtung spiegelt sich in der bisherigen Beurteilungspraxis der Kartellbehörden wider. Seit dem Jahre 2008 ist in Deutschland eine Tendenz zur zumindest partiellen Würdigung der Verbundenheit von Märkten über indirekte Netzeffekte zu beobachten, wohingegen der TMT in den USA bisher keine kartellrechtliche Relevanz beigemessen wird. Als Ursache für diese Unterschiede können verschiedene Aspekte diskutiert werden:

Ein Grund könnte in den konkreten Anwendungsproblemen bestehen. Insbesondere die USamerikanischen Kartellbehörden greifen für die Marktabgrenzung überwiegend auf den

40 Bundeskartellamt, 2016, S. 11. 
SSNIP-Test zurück, wie es in den Merger Guidelines vorgesehen ist. Diese sind zwar rechtlich nicht verbindlich, bilden dennoch die theoretische Grundlage der Zusammenschlussprüfung. Die sich bei seiner Übertragung auf Medienmärkte ergebenden Probleme wurden unter Punkt 2.3 beschrieben. Einige Autoren lehnen die Möglichkeit der Anpassung gänzlich ab, andere empfehlen die Verwendung der Summe der von der Plattform gesetzten Preise zur Beurteilung der Änderung des Preisniveaus durch den Zusammenschluss. Ein wissenschaftlicher Konsens wurde bisher nicht gefunden. ${ }^{41}$ Aufgrund der langjährigen Praxis könnte eine Abkehr vom (herkömmlichen) SSNIP-Test sowohl mit methodischen Problemen als auch mit Gegenwehr der Kartellbehörde(n) verbunden sein. Das in Deutschland vorherrschende Bedarfsmarktkonzept könnte im Gegenzug eventuell leichter an plattformspezifische Besonderheiten anzupassen sein.

Auf der Stufe der Prüfung der Wettbewerbswirkungen des Zusammenschlusses lässt sich in den Merger Guidelines eine starke Ausrichtung auf den HHI und die Preisentwicklung beobachten. Die für den HHI vorgegebenen Grenzen wurden mit der Revidierung der Merger Guidelines im Jahre 1982 festgeschrieben und dürften von den Kartellbehörden inzwischen als empirisch gesichert und bewährt angesehen werden. Die veränderte Bewertung der Marktkonzentration lässt sich damit nicht vereinbaren. Der Einbezug der TMT würde eine wesentliche Abkehr von der bisherigen Sichtweise bedeuten, insbesondere da neue Maßstäbe für die Bewertung der Marktstellung von Unternehmen entwickelt werden müssten. Relativiert wird diese Argumentation indes durch die tatsächliche Praxis der Kartellbehörden, die eine weniger restriktive Auslegung der HHI-Schwellenwerte der Merger Guidelines im Zeitablauf erkennen lässt. Vielmehr stellen die Marktanteile und die Marktkonzentration auch in den USA lediglich den Ausgangspunkt für die Betrachtung dar. Eine Relativierung durch andere Faktoren ist ebenso wie in Deutschland möglich. ${ }^{42}$

Darüber hinaus ist die Beurteilung der Wohlfahrtseffekte eines Zusammenschlusses auf Plattformmärkten mit der Notwendigkeit der Bestimmung der (relativen) Stärke der indirekten Netzeffekte verbunden. Einheitliche Verfahren existieren hierfür derzeit nicht. Da die Stärke der Netzeffekte und deren disziplinierende Wirkung zur Wahrung der eigenen Marktstellung geschätzt werden müssen, besteht grundsätzlich die Gefahr von Fehlurteilen, wenn falsche

41 Vgl. Filistrucchi et al., 2014, S. 331, Emch/Thompson, 2006, S. 17, Filistrucchi, 2008, S. 15 f., Evans, 2011 , S. 149. Kritisch zur Anwendung des SSNIP-Tests auf die Summe aus beiden Preisen vgl. Kehder, 2013, S. 80 f.

42 Vgl. hierzu ausführlicher Petrasincu, 2009, S. 89 ff. 
Annahmen getroffen werden. So könnte ein Zusammenschluss, der infolge von Netzeffekten freigegebenen wurde, nach Vollzug entgegen der Prognose wettbewerbsschädliche Wirkungen zeigen. Zwar muss Marktmacht auf Plattformmärkten nicht zwingend wohlfahrtsschädigend sein, missbräuchliches Verhalten kann jedoch ebenso wenig generell ausgeschlossen werden. ${ }^{43}$ Die grundsätzliche Anerkennung wohlfahrtssteigernder Effekte durch steigende Marktkonzentration lässt sich anscheinend mit dem derzeitigen Wettbewerbsverständnis der US-amerikanischen Kartellbehörden nicht vereinbaren. Zumindest deuten die vorstehenden Ergebnisse auf eine geringere Bereitschaft zur Abkehr von der klassischen Sichtweise hin als in Deutschland.

Ein weiterer Unterschied könnte in der Ausgestaltung und Auslegung des jeweiligen Kartellrechts bestehen. Die Zielstellung und Wettbewerbskonzeption der deutschen und der USamerikanischen Zusammenschlusskontrolle weisen zunächst eine Reihe von Gemeinsamkeiten auf. Beide orientieren sich wesentlich am Konzept des funktionsfähigen Wettbewerbs und verstehen Wettbewerb als ein Mittel zur Erreichung gesamtwirtschaftlicher Ziele. Ferner zeigen beide eine deutliche Orientierung an der Marktstruktur. Sowohl der Sherman- und Clayton Act als auch das GWB zielen auf die Verhinderung wirtschaftlicher Macht, die es Unternehmen ermöglicht, den Wettbewerb erheblich zu behindern, verfälschen oder gar auszuschalten. ${ }^{44}$ Hinsichtlich der Auslegung der gesetzlichen Regelungen ergeben sich hingegen gewisse Unterschiede. Beispielsweise war in den USA eine stärkere Ausrichtung an den Lehren der Harvard und der Chicago School als in Deutschland zu erkennen. Darüber hinaus unterliegt die kartellrechtliche Praxis der ATD und der FTC einem stärkeren politischen Einfluss als die des Bundeskartellamts. Die Auslegung der Regelungen des Sherman und des Clayton Acts ließen in der Vergangenheit eine deutliche Ausrichtung an die wirtschaftspolitische Leitlinien des jeweils amtierenden Präsidenten erkennen, nicht zuletzt aufgrund seines Einflusses auf die Besetzung der Kartellbehörden. Nach der restriktiven Zusammenschlusskontrolle kam es in den 1980ern unter Reagan zu einer deutlichen Deregulierungspolitik mit Lockerungen in der Durchsetzung der Zusammenschlusskontrolle, wohingegen in den sich anschließenden Amtszeiten von Bush Senior und Clinton ein erneuter Anstieg kartellrechtlicher Prüfungen zu verzeichnen war. ${ }^{45}$ Die Kartellrechtspraxis im Untersuchungszeitraum, der sich weitgehend auf

\footnotetext{
43 Vgl. Europäische Kommission, 2009, S. 17.

44 Vgl. Christiansen, 2000, S. $51 \mathrm{ff}$.

45 Ausführlich hierzu vgl. Christiansen, 2000, S. 21, Bundeskartellamt, 2001, S. 8. Zum Einfluss der Politik auf das Kartellrecht vgl. Scherer, 2007, S. 9 f.
} 
die Amtszeiten von Bush Junior und Obama erstreckte, war wiederum durch eine weniger restriktive Durchsetzung der Zusammenschlusskontrolle geprägt. In den vorliegenden Ergebnissen spiegelt sich dies in der Vielzahl (unter Auflagen) freigegebener Verfahren wider. Insofern orientiert sich die US-amerikanische Antitrustpolitik stärker an der Politik als die deutsche, was sich wiederum auf die Bereitschaft zur Integration neuer wissenschaftlicher Erkenntnisse ausgewirkt haben kann. Zudem wurde ein Großteil der angemeldeten Zusammenschlussvorhaben in den USA unter Auflagen freigegeben, während Deutschland im Untersuchungszeitraum eine vergleichsweise restriktivere Kartellechtspraxis aufwies. Die Würdigung weiterer theoretischer Argumente für eine Freigabe könnte für ATD und FTC somit von geringerer Bedeutung gewesen sein.

Inwiefern eine wie in Deutschland angestrebte Erweiterung des Prüfungskatalogs automatisch zu einer stringenteren Anwendung der TMT führt, bleibt abzuwarten, als die beschriebenen praktischen Anwendungsprobleme, wie beispielsweise die Bestimmung der (relativen) Stärke der Netzeffekte fortbestehen. Ungeachtet des Fortschritts der Aufnahme der TMT in das Kartellrecht besteht somit sowohl in Deutschland als auch den USA grundsätzlicher Handlungsbedarf auf wissenschaftlicher Ebene hinsichtlich der Operationalisierung der theoretischen Erkenntnisse.

\section{Schlussbemerkungen}

Im vorliegenden Beitrag wurde die kartellrechtliche Praxis der US-amerikanischen und deutschen Wettbewerbsbehörden im Zeitraum von 1999 bis 2016 vorgenommen. Die Analyse der Zusammenschlussfälle von Medienunternehmen lässt entgegen der theoretischen Bedeutsamkeit der Würdigung plattformspezifischer Besonderheiten eine im Zeitablauf zunehmende praktische Relevanz der Theorie mehrseitiger Märkte lediglich in der deutschen Zusammenschlusskontrolle erkennen. Während ATD und FTC lediglich in zwei der untersuchten 30 Fälle auf die Verbundenheit der Märkte eingingen, ist diese in Deutschland seit 2008 häufiger thematisiert worden. Allerdings beschränkten sich die Kartellbehörden auch hier meist auf einzelne Elemente. Eine durchgängige Anwendung der Theorie findet bisher noch nicht statt. Ebenso wie in den USA wird somit ein Großteil der Zusammenschlussvorhaben nach den Regeln einseitiger Märkte geprüft. Die theoretischen Forderungen und die praktische Umsetzung fallen somit noch auseinander. 
Ein Einbezug der neuen wissenschaftlichen Erkenntnisse in die kartellrechtlichen Gesetzesgrundlagen, wie er vom deutschen Gesetzgeber derzeit vollzogen wird, ist vor diesem Hintergrund prinzipiell zu begrüßen. Größere Bedeutung als der rechtlichen Verankerung sollte indes der Anpassung und Weiterentwicklung des bestehenden Instrumentariums zur Marktabgrenzung sowie zur Beurteilung der Marktstellung und des Marktverhaltens von Plattformen beigemessen werden. Insbesondere der in der US-amerikanischen Antitrustpolitik gebräuchliche SSNIP-Test ist zur Definition des relevanten Marktes, wenn überhaupt, lediglich unter Anpassungen anwendbar. Darüber hinaus fehlen bisher geeignete Verfahren zur verlässlichen Bestimmung der Höhe der indirekten Netzeffekte. Neben politischen Einflüssen auf die Zusammenschlusskontrolle lassen sich diese Probleme bei der Operationalisierung der Theorie mehrseitiger Marktplätze als ein wesentlicher Grund für ihre bisher geringe praktische Relevanz in den USA und die nicht stringente Anwendung in Deutschland vermuten.

\section{Literaturverzeichnis}

American Antitrust Institute (AAl, 2015), Comments of the American Antitrust Institute, FTC File No. P143100, Washington D.C. 2015.

Anderson, Simon P./McLaren, John, Media Mergers and Media Bias with Rational Consumers, in: Journal of the European Association, Vol. 10, 4/2012, S. 831 - 859.

Armstrong, Mark, Competition in Two-Sided Markets, in: The RAND Journal of Economics, Vol. $37,3 / 2006$, S. $668-691$.

Auer, Dirk/Petit, Nicolas, Two-Sided Markets and the Challenge of Turning Economic Theory into Competition Policy, University of Liege, Working Paper, Lüttich 2015.

Baker, Jonathan B., Market Power in the U.S. Economy Today, Discussion Paper, The Washington Center for Equitable Growth, Washington D.C. 2017.

Brenkers, Randy/Verboven, Frank, Market Definition with Differentiated Products-Lessons from the Car Market, in: Antitrust: Theory and Evidence, Cambridge 2007, S. 153 - 186.

Budzinski, Oliver, Europaïsche Medienmärkte: Wettbewerb, Meinungsvielfalt und kulturelle Vielfalt, University of Southern Denmark, Working Paper, Esbjerg 2009.

Budzinski, Oliver, Wettbewerbsregeln für das Digitale Zeitalter? Die Ökonomik personalisierter Daten, Verbraucherschutz und die 9. GWB-Novelle, in: List Forum, 43. Jg., 3/2017, S. $221-249$. 
Budzinski, Oliver/Kuchinke, Björn, Modern Industrial Organization Theory of Media Markets and Competition Policy Implications, Ilmenau Economics Discussion Papers Nr. 115, TU Ilmenau, Institut für Volkswirtschaftslehre, September 2018.

Budzinski, Oliver/Lindstädt, Nadine, Neuere Entwicklungen in der Medienökonomik: Das Konzept der mehrseitigen Märkte, in: Wirtschaftswissenschaftliches Studium, 39. Jg., 9/2010, S. $436-443$.

Budzinski, Oliver/Stöhr, Annika, Competition policy reforms in Europe and Germany - institutional change in the light of digitization, in : European Competition Journal, 15:1, 1554, DOI: 10.1080/17441056.2018.1555942, 2019.

Budzinski, Oliver/Wacker, Katharina, The Prohibition of the Proposed Springer-ProSiebenSat.1 Merger: How Much Economics in German Merger Control?, in: Journal of Competition Law and Economics?, Vol. 3, 2/2007, S. $281-306$.

Bundeskartellamt, Pressemitteilung vom 02.03.2016 „Bundeskartellamt eröffnet Verfahren gegen Facebook wegen Verdachts auf Marktmachtmissbrauch durch Datenschutzverstöße“. Abrufbar unter: https://www.bundeskartellamt.de/SharedDocs/Meldung/DE/Pressemitteilungen/2016/02_03_2016_Facebook.html;jsessionid=6E24C416913E04144BF758D1DEF6760E.1_cid371?nn=3591568.

Bundeskartellamt, Stellungnahme des Bundeskartellamts zum Regierungsentwurf zur 9. GWBNovelle, Ausschussdrucksache 18(9)1093 vom 19. Januar 2017, Bonn 2017.

Christiansen, Arndt, Ein Vergleich zwischen europäischer und US-amerikanischer Fusionskontrolle, Hamburg 2000 (Zugl.: Marburg, Univ., Diplomarbeit, 2000).

Deutscher Bundestag, Entwurf eines Neunten Gesetzes zur Änderung des Gesetzes gegen Wettbewerbsbeschränkungen, Drucksache 18/10207 vom 07.11.2016, Berlin 2016.

Dewenter, Ralf, Two-Sided Markets, in: MedienWirtschaft, 3. Jg., 2/2006, S. $57-62$.

Dewenter, Ralf, Crossmediale Fusionen und Meinungsvielfalt, Helmut-Schmidt-Universität Hamburg, Discussion Paper No. 65, Hamburg 2007.

Dewenter, Ralf/Haucap, Justus, Wettbewerb als Aufgabe und Problem auf Medienmärkten, in: Wenzel, Dirk, Medienökonomik: Theoretische Grundlagen und ordnungspolitische Gestaltungsalternative, Stuttgart 2009, S. 35 - 73.

Dewenter, Ralf/Haucap, Justus/Wenzel, Tobias, Semi-Collusion in Media Markets, in: International Review of Law and Economics, 31. Jg., 2/2011, S. $92-98$. 
Dewenter, Ralf/Kaiser, Ulrich, Fusionen auf dem Printmedienmarkt aus wirtschaftstheoretischer Sicht, in: Vierteljahrshefte zur Wirtschaftsforschung, 74. Jg., 3/2005, S. 51 - 63.

Dewenter, Ralf/Kaiser, Ulrich, Horizontale Fusionen auf zweiseitigen Märkten am Beispiel von Printmedien, in: Perspektiven für Wirtschaftspolitik, 7. Jg., 3/2006, S. 335 - 353.

Dewenter, Ralf/Rösch, Jürgen/Terschüren, Anna, Abgrenzung zweiseitiger Märkte am Beispiel von Internetsuchmaschinen, Helmut Schmidt Universität Hamburg, Diskussionspapier Nr. 151, Hamburg 2014.

Emch, Eric/Thompson, T. Scott, Market Definition and Market Power in Payment Card Networks, Economic Analysis Group Discussion Paper, Washington 2006.

Europäische Kommission, Round Table on Two-Sided Markets: Note by the Delegation of the European Commission, DAF/COMP/WD (2009)69, Brüssel 2009.

Eurostat, NACE Rev. 2: Statistische Systematik der Wirtschaftszweige in der Europäischen Gemeinschaft, Methodologies and Working papers, Luxembourg 2008.

Evans, David S. (Evans, 2003a), Some Empirical Aspects of Multi-Sided Platform Industries, in: Review of Network Economics, Vol. 2, 3/2003, S. $191-209$.

Evans, David S., (Evans, 2003b), The Antitrust Economics of Multi-Sided Platform Markets, in: Yale Journal on Regulation, 2/2003, S. $325-381$.

Evans, David S., Competition and Regulatory Policy for Multi-Sided Platforms with Application to the Web Economy, University of Chicago Law School, Working Paper, Cambridge 2008.

Evans, David S., The Web Economy, Two-Sided Markets and Competition Policy, University of Chicago Law School; University College London, Working Paper, 2010.

Evans, David S., The Anitrust Economics of Free, Competition Policy International, Vol. 7, 1/2011, S. $70-89$.

Evans, David S.,/Schmalensee, Richard, The Industrial Organization of Markets with Two-Sided Platforms, in: Competition Policy International, Vol. 3, 1/2007, S. 150 - 179.

Evans, David S.,/Schmalensee, Richard, The Antitrust Analysis of Multi-Sided Platform Businesses, National Bureau of Economic Research, Working Paper, Cambridge 2012.

Filistrucchi, Lapo, A SSNIP Test for Two-Sided Markets: Some Theoretical Considerations, Discussion Paper, 2008. 
Filistrucchi, Lapo/Geradin, Damien/Damme, Eric van/Affeldt, Pauline (Filistrucchi et al., 2014), Market Definition in Two-Sides Markets: Theory and Practice, in: Journal of Competition Law \& Economics, Vol. 10, 2/2014, S. $293-339$.

Gustafsson, Karl Erik, The Circulation Spiral and the Principle of Household Coverage, in: Scandinavian Economic History Review, Vol. 26, 1/1978, S. 1 - 14.

Hamelmann, Lisa/Haucap, Justus, Kartellrecht und Wettbewerbspolitik für Online-Plattformen, DICE Ordnungspolitische Perspektiven, Nr. 78, Düsseldorf 2015.

Hass, Berthold H., Größenvorteile von Medienunternehmen: Eine kritische Würdigung der Anzeigen-Auflagen-Spirale, in: MedienWirtschaft, Sonderheft 2007, S. $70-78$.

Haucap, Justus/Wenzel, Tobias, Ist eBay unbestreitbar ein nicht bestreitbares Monopol?, Monopolisierungsgefahren und Regulierungsbedarf bei Online-Marktplätzen, in: Kruse, Jörn/Dewenter, Ralf (Hrsg.), Wettbewerbsprobleme im Internet, Baden-Baden 2009, S. $7-34$.

Katz, Michael L./Shapiro, Carl, Network Externalities, Competition, and Compatibility, in: The American Economic Review, Vol. 75, 3/1985, S. 424 - 440.

Kaywaty, Henry J./Tyler, Cleve B., Market Definition: Achieving an Integrated Analysis, in: Antitrust Bulletin, Vol. 59, 3/2014, S. $667-685$.

Kehder, Christiane, Konzepte und Methoden der Marktabgrenzung und ihre Anwendung auf zweiseitige Märkte, Baden-Baden 2013 (Zugl.: Hamburg, Helmut-Schmidt-Univ., Diss., 2013).

Klein, Benjamin/Lerner, Andreas V./Murphy, Kevin M., Plache, Lacey L. (Klein et al., 2006), Competition in Two-Sided Markets: The Antitrust Economics of Payment Card Interchange Fees, in: Antitrust Law Journal, 3/2006, S. 571 - 626.

Lindstädt, Nadine, Multisided Media Markets: Applying the Theory of Multisided Markets to Media Markets, University of Southern Denmark, Workung Paper No. 96, Esbjerg 2009. Monopolkommission, Fünften Hauptgutachten der Monopolkommission 1892/1983, in: Deutscher Bundestag, Drucksache 10/1791, Bonn 1984.

Motta, Massimo, Competition Policy: Theory and Practice, Cambridge u. a. 2004.

Nussberger, Ulrich, Dynamik der Zeitung, Stuttgart 1961.

Petrasincu, Alex, Horizontale, vertikale und konglomerate Zusammenschlüsse in der europäischen und amerikanischen Fusionskontrolle, Baden-Baden 2009 (Zugl.: Münster (Westf.), Univ., Diss., 2009). 
Rochet, Jean-Charles/Tirole, Jean, Platform Competition in Two-Sided Markets, in: Journal of the European Economic Association, Vol. 1, 4/2003, S. 990 - 1029.

Roson, Roberto, Two-Sided Markets: A Tentative Survey, in: Review of Network Economics, Vol. 4, 2/2005, S. $142-160$.

Rysman, Marc, The Economics of Two-Sided Markets, in: Journal of Economic Perspectives, Vol. 23, 3/2009, S. $125-143$.

Scherer F. M., How Conservative Economics Has Influenced Antitrust, Harvard University, Faculty Research Working Paper Series, Working Paper RWP07-027, Boston 2007.

Schmalensee, Richard, Payment Systems and Interchange Fees, in: The Journal of Industrial Economics, Vol. 50, 2/2002, S. $103-122$.

U.S. Department of Justice/Federal Trade Commission (DoJ/FTC, 2010), Horizontal Merger Guidelines, Washington D.C. 2010.

Weisman, Dennis L., Market Concentration, Multi-Market Participation and Mergers in Network Industries, in: Review of Network Economics, Vol. 4, 2/2005, S. $129-141$.

Weyl, Glen, A Price Theory of Multi-Sided Platforms, in: American Economic Review, Vol. 100, 4/2010, S. $1642-1672$.

White, Lawrence J., Market Definition and Market Power in Payment Card Networks: Some Comments and Considerations, New York University, Stern School of Business Working Paper, New York 2006.

Wright, Julian, One-Sided Logic in Two-Sided Markets, in: Review of Network Economics, Vol. $3,1 / 2004$, S. $44-64$. 


\section{Diskussionspapiere aus dem Institut für Volkswirtschaftslehre der Technischen Universität IImenau}

Nr. 69 Budzinski, Oliver: Empirische Ex-Post Evaluation von wettbewerbspolitischen Entscheidungen: Methodische Anmerkungen, Januar 2012.

Nr. $70 \quad$ Budzinski, Oliver: The Institutional Framework for Doing Sports Business: Principles of EU Competition Policy in Sports Markets, January 2012.

Nr. 71 Budzinski, Oliver; Monostori, Katalin: Intellectual Property Rights and the WTO, April 2012.

Nr. $72 \quad$ Budzinski, Oliver: International Antitrust Institutions, Juli 2012.

Nr. 73 Lindstädt, Nadine; Budzinski, Oliver: Newspaper vs. Online Advertising - Is There a Niche for Newspapers in Modern Advertising Markets?

Nr. 74 Budzinski, Oliver; Lindstädt, Nadine: Newspaper and Internet Display Advertising Co-Existence or Substitution?, Juli 2012b.

Nr. 75 Budzinski, Oliver: Impact Evaluation of Merger Control Decisions, August 2012.

Nr. 76 Budzinski, Oliver; Kuchinke, Björn A.: Deal or No Deal? Consensual Arrangements as an Instrument of European Competition Policy, August 2012.

Nr. 77 Pawlowski, Tim, Budzinski, Oliver: The (Monetary) Value of Competitive Balance for Sport Consumers, Oktober 2012.

Nr.78 Budzinski, Oliver: Würde eine unabhängige europäische Wettbewerbsbehörde eine bessere Wettbewerbspolitik machen?, November 2012.

Nr. 79 Budzinski, Oliver; Monostori, Katalin; Pannicke, Julia: Der Schutz geistiger Eigentumsrechte in der Welthandelsorganisation - Urheberrechte im TRIPS Abkommen und die digitale Herausforderung, November 2012.

Nr. 80 Beigi, Maryam H. A.; Budzinski, Oliver: On the Use of Event Studies to Evaluate Economic Policy Decisions: A Note of Caution, Dezember 2012.

Nr. 81 Budzinski, Oliver; Beigi, Maryam H. A.: Competition Policy Agendas for Industrializing Countries, Mai 2013.

Nr. 82 Budzinski, Oliver; Müller, Anika: Finanzregulierung und internationale Wettbewerbsfähigkeit: der Fall Deutsche Bundesliga, Mai 2013.

Nr. 83 Doose, Anna Maria: Methods for Calculating Cartel Damages: A Survey, Dezember 2013. 
Nr. 84 Pawlowski, Tim; Budzinski, Oliver: Competitive Balance and Attention Level Effects: Theore-tical Considerations and Preliminary Evidence, März 2014.

Nr. 85 Budzinski, Oliver: The Competition Economics of Financial Fair Play, März 2014.

Nr. 86 Budzinski, Oliver; Szymanski, Stefan: Are Restrictions of Competition by Sports Associations Horizontal or Vertical in Nature?, März, 2014.

Nr. 87 Budzinski, Oliver: Lead Jurisdiction Concepts Towards Rationalizing Mul-tiple Competition Policy Enforcement Procedures, Juni 2014.

Nr. 88 Budzinski, Oliver: Bemerkungen zur ökonomischen Analyse von Sicherheit, August 2014.

Nr. 89 Budzinski, Oliver; Pawlowski, Tim: The Behavioural Economics of Competitive Balance: Implications for League Policy and Championship Management, September 2014.

Nr. 90 Grebel, Thomas; Stuetzer, Michael: Assessment of the Environmental Performance of European Countries over Time: Addressing the Role of Carbon

Nr. 91 Emam, Sherief; Grebel, Thomas: Rising Energy Prices and Advances in Renewable Energy Technologies, July 2014.

Nr. 92 Budzinski, Oliver; Pannicke, Julia: Culturally-Biased Voting in the Eurovision Song Contest: Do National Contests Differ?, December 2014.

Nr. 93 Budzinski, Oliver; Eckert, Sandra: Wettbewerb und Regulierung, März 2015.

Nr.94 Budzinski, Oliver; Feddersen, Arne: Grundlagen der Sportnachfrage: Theorie und Empirie der Einflussfaktoren auf die Zuschauernachfrage, Mai 2015.

Nr.95 Pannicke, Julia: Abstimmungsverhalten im Bundesvision Song Contest: Regionale Nähe versus Qualität der Musik, Oktober 2015.

Nr.96 Budzinski, Oliver; Kretschmer, Jürgen-Peter: Unprofitable Horizontal Mergers, External Effects, and Welfare, October 2015.

Nr. 97 Budzinski, Oliver; Köhler, Karoline Henrike: Is Amazon The Next Google?, October 2015.

Nr. 98 Kaimann, Daniel; Pannicke, Julia: Movie success in a genre specific contest: Evidence from the US film industry, December 2015.

Nr. 99 Pannicke, Julia: Media Bias in Women's Magazines: Do Advertisements Influence Editorial Content?, December 2015. 
Nr. 100 Neute, Nadine; Budzinski, Oliver: Ökonomische Anmerkungen zur aktuellen Netzneutralitätspolitik in den USA, Mai 2016.

Nr. 101 Budzinski, Oliver; Pannicke, Julia: Do Preferences for Pop Music Converge across Countries? - Empirical Evidence from the Eurovision Song Contest, Juni 2016.

Nr. 102 Budzinski, Oliver; Müller-Kock, Anika: Market Power and Media Revenue Allocation in Professonal Sports: The Case of Formula One, Juni 2016.

Nr.103 Budzinski, Oliver: Aktuelle Herausforderungen der Wettbewerbspolitik durch Marktplätze im Internet, September 2016.

Nr. 104 Budzinski, Oliver: Sind Wettbewerbe im Profisport Rattenrennen?, Februar 2017.

Nr. 105 Budzinski, Oliver; Schneider, Sonja: Smart Fitness: Ökonomische Effekte einer Digitalisierung der Selbstvermessung, März 2017.

Nr. 106 Budzinski, Oliver; Pannicke, Julia: Does Popularity Matter in a TV Song Competition? Evidence from a National Music Contest, April 2017.

Nr. 107 Budzinski, Oliver; Grusevaja, Marina: Die Medienökonomik personalisierter Daten und der Facebook-Fall, April 2017.

Nr. 108 Budzinski, Oliver: Wettbewerbsregeln für das Digitale Zeitalter - Die Ökonomik personalisierter Daten, Verbraucherschutz und die 9.GWB-Novelle, August 2017.

Nr. 109 Budzinski, Oliver: Four Cases in Sports Competition Policy: Baseball, Judo, Football, and Motor Racing, September 2017.

Nr. 110 Budzinski, Oliver: Market-internal Financial Regulation in Sports as an Anticompetitive Institution, October 2017.

Nr. 111 Bougette, Patrice; Budzinski, Oliver; Marty, Frédéric: EXPLOITATIVE ABUSE AND ABUSE OF ECONOMIC DEPENDENCE: WHAT CAN WE LEARN FROM THE INDUSTRIAL ORGANIZATION APPROACH?, December 2017.

Nr. 112 Budzinski, Oliver; Gaenssle, Sophia: The Economics of Social Media Stars: An Empirical Investigation of Stardom, Popularity, and Success on YouTube, Januar 2018.

Nr. 113 Gaenssle, Sophia; Budzinski, Oliver; Astakhova Daria: Conquering the Box Office: Factors, influencing Success of International Movies in Russia, Mai 2018.

Nr. 114 Budzinski, Oliver; Stöhr, Annika: Die Ministererlaubnis als Element der deutschen Wettbewerbsordnung: eine theoretische und empirische Analyse, Juli 2018. 
Nr. 115 Budzinski, Oliver; Kuchinke, Björn A.: Modern Industrial Organization Theory of Media Markets and Competition Policy Implications, September 2018.

Nr. 116 Budzinski, Oliver; Lindstädt-Dreusicke, Nadine: The New Media Economics of Video-on-Demand Markets: Lessons for Competition Policy, Oktober 2018.

Nr. 117 Budzinski, Oliver; Stöhr, Annika: Competition Policy Reform in Europe and Germany - Institutional Change in the Light of Digitization, November 2018.

Nr. 118 Budzinski, Oliver; Noskova, Victoriia; Zhang, Xijie: The Brave New World of Digital Personal Assistants: Benefits and Challenges from an Economic Perspective, December 2018.

Nr. 119 Bougette, Patrice; Budzinski, Oliver \& Marty, Frédéric: EXPLOITATIVE ABUSE AND ABUSE OF ECONOMIC DEPENDENCE: WHAT CAN WE LEARN FROM AN INDUSTRIAL ORGANIZATION APPROACH? [Updated Version 2018], December 2018.

Nr. 120 Bartelt, Nadja: Bundling in Internetmärkten - Ökonomische Besonderheiten, Wettbewerbseffekte und Regulierungsimplikationen, Dezember 2018.

Nr. 121 Budzinski, Oliver; Feddersen, Arne: Measuring Competitive Balance in Formula One Racing, März 2019.

Nr. 122 Budzinski, Oliver; Kohlschreiber, Marie; Kuchinke, Björn A. \& Pannicke, Julia: Does Music Quality Matter for Audience Voters in a Music Contest, März 201

Nr. 123 Gaenssle, Sophia \& Budzinski, Oliver: Stars in Social Media: New Light Through Old Windows?, April 2019.

Nr. 124 Stöhr, Annika; Budzinski, Oliver: Ex-post Analyse der Ministererlaubnis-Fälle Geminwohl durch Wettbewerbsbeschränkungen?, April 2019.

Nr. 125 Budzinski, Oliver; Lindstädt-Dreusicke, Nadine: The New Media Economics of Video-on-Demand Markets: Lessons for Competition Policy (Updated Version), May 2019.

Nr. 126 Stöhr, Annika; Noskova, Victoriia; Kunz-Kaltenhäuser, Philipp; Gaenssle, Sophia \& Budzinski, Oliver: Happily Ever After? - Vertical and Horizontal Mergers in the U.S. Media Industry, June 2019.

Nr.127 Budzinski, Oliver; Stöhr, Annika: Der Ministererlaubnis-Fall Miba/Zollern: Europäische Champions statt Wettbewerb?, Juni 2019.

Nr. 128 Budzinski, Oliver; Gaenssle, Sophia \& Kunz-Kaltenhäuser, Philipp: How Does Online Streaming Affect Antitrust Remedies to Centralized Marke-ting? The Case of European Football Broadcasting, Rights, July 2019. 
Nr. 129 Budzinski, Oliver; Haucap, Justus: Kartellrecht und Ökonomik: Institutions Matter!, July 2019.

Nr. 130 Budzinski, Oliver \& Stöhr, Annika: Public Interest Considerations in European Merger Control Regimes, August 2019.

Nr. 131 Gaenssle, Sophia; Budzinski, Oliver \& Astakhova: Daria: Conquering the Box Office: Factors Influencing Succes of International Movies in Russia (Update), October 2019.

Nr. 132 Stöhr, Annika; Budzinski, Oliver \& Jasper, Jörg: Die Neue E.ON auf dem deutschen Strommarkt - Wettbewerbliche Auswirkungen der innogy-Übernahme, November 2019.

Nr. 133 Budzinski, Oliver; Grebel, Thomas; Wolling, Jens \& Zhang, Xijie: Drivers of Article Processing Charges in Open Access, December 2019.

Nr. 134 Gaenssle, Sophia; Kuchinke, Björn, A.: Die Hörspielserie „Die drei ???“ - Der wirtschaftliche Erfolg und Gründe dafür, Januar 2020.

Nr. 135 Budzinski, Oliver: The Economics of International Competition Policy: New Challenges in the Light of Digitization?, January 2020. 